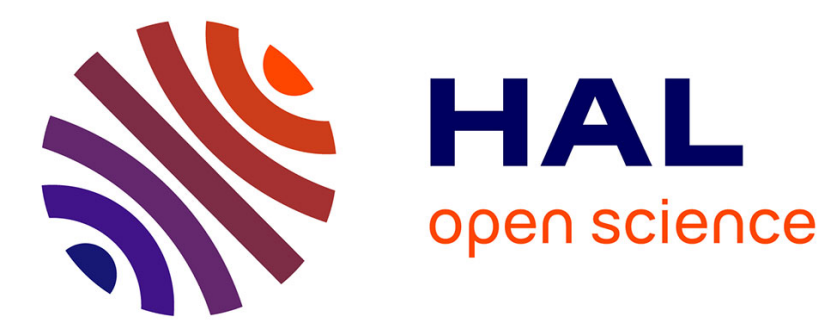

\title{
Magnetic field approaches in dc thermal plasma modelling
}

Pierre Freton, Jean Jacques Gonzalez, Mathieu Masquère, Frank Reichert

\section{To cite this version:}

Pierre Freton, Jean Jacques Gonzalez, Mathieu Masquère, Frank Reichert. Magnetic field approaches in dc thermal plasma modelling. Journal of Physics D: Applied Physics, 2011, 44 (34), pp.345202. 10.1088/0022-3727/44/34/345202 . hal-00646921

\section{HAL Id: hal-00646921 \\ https://hal.science/hal-00646921}

Submitted on 1 Dec 2011

HAL is a multi-disciplinary open access archive for the deposit and dissemination of scientific research documents, whether they are published or not. The documents may come from teaching and research institutions in France or abroad, or from public or private research centers.
L'archive ouverte pluridisciplinaire HAL, est destinée au dépôt et à la diffusion de documents scientifiques de niveau recherche, publiés ou non, émanant des établissements d'enseignement et de recherche français ou étrangers, des laboratoires publics ou privés. 


\title{
Magnetic field approaches in DC thermal plasma modelling
}

\author{
P. Freton ${ }^{1}$, J.J. Gonzalez ${ }^{1}$, M. Masquère ${ }^{1}$, Frank Reichert ${ }^{2}$ \\ ${ }^{1}$ Laplace, UMR 5213 CNRS-UPS-INP, Université Paul Sabatier, bat 3R2, 118 rte de Narbonne, 31062 Toulouse \\ Cedex, France \\ ${ }^{2}$ Siemens AG, E T HP CB PLM R\&D B PB, Nonnendammallee 104, 13629 Berlin, Germany \\ frank.reichert@siemens.com \\ Email: pierre.freton@laplace.univ-tlse.fr
}

\begin{abstract}
The self-induced magnetic field has an important role in thermal plasma configurations generated by electric arcs as it generates velocity through Lorentz forces. In the models a good representation of the magnetic field is thus necessary. Several approaches exist to calculate the self-induced magnetic field such as the Maxwell - Ampere formulation, the vector potential approach combined with different kinds of boundary conditions or the Biot \& Savart formulation. The calculation of the self-induced magnetic field is alone a difficult problem and only few articles of the thermal plasma community speak on this subject. In the present study different approaches with different boundary conditions are applied on two geometries to compare the methods and their limitations. The calculation time is also one of the criteria for the choice of the method and a compromise must be found between method precision and computation time. The study shows the importance of the current carrying path representation in the electrode on the deduced magnetic field. The best compromise consists of using the Biot \& Savart formulation on the walls and/or edges of the calculation domain to determine the boundary conditions and to solve the vector potential in a 2D system. This approach provides results identical to those obtained using the Biot \& Savart formulation over the entire domain but with a considerable decrease of calculation time.
\end{abstract}

Keywords : - Thermal plasma - Electric arc, Magnetic field boundary conditions 


\section{Introduction}

The aim of this study is to asses various methods used to determine the contribution of the self-induced magnetic field created by the presence of the electric arc in thermal plasma modelling.

Numerous formulations have been proposed over the years to estimate the selfinduced magnetic field in DC or AC thermal plasma configurations. Chronologically, the first formulation, in the eighties, was the Maxwell - Ampere (M.A.) form which has been used in two-dimensional (2D) modelling in numerous articles such as Hsu [1] or Lowke [2]. It is very convenient in $2 \mathrm{D}$ on a structured grid but not really suited to an unstructured one. Moreover, it cannot be used in three dimensional (3D) modelling and due to the evolution of the models from $2 \mathrm{D}$ to $3 \mathrm{D}$, alternatives formulations have been chosen in the nineties.

In order to estimate the self-induced magnetic field in 3D cases, the best way should be to use the Biot \& Savart (B\&S) equation. Some authors have chosen this formulation [3-5]. Nevertheless, for each point of the calculation domain it requires volume integration which can consume more than $90 \%$ of the computational time. Instead of using the $\mathrm{B} \& \mathrm{~S}$ formulation, almost all authors use the potential vector (PV) approach ([6-11] for example). The advantage of this formulation is that each vector potential component can be written as one equation having the same form as the generalized equation used as for the fluid description.

Nevertheless, the use of the PV formulation implies that care should be taken in the choice of boundary conditions. Indeed, the potential vector should be equal to zero at infinity. This specific condition cannot be reached in all models (problems in constricted parts and necessity to assume a large calculation domain). This point has been studied by some authors in RF plasmas configurations where people propose to calculate the magnetic field in an extended domain [12][13] or by B\&S equations [12]. In AC/DC, in order to overcome this problem, the authors very often consider a null flux as boundary condition for vector potential components at the modelled geometry edges [9-10] or a value equal to zero if the boundary is far from the arc [6-8]. Very few authors, as He-Ping Li [11], propose, only for three dimensional DC configurations the use of Biot \& Savart equation as a boundary condition for potential vector.

In this article, we want to point out that the MA formulation can be used only in the event of zero axial gradient of the current density. We would show that the use of the potential vectors without good boundary conditions can lead to wrong results and also to 
conclude on an alternative solution by studying a mixed method based on B\&S and P.V. formulations.

In order to present the study and the developments, a simple $2 \mathrm{D}$ configuration of a transferred arc burning in argon at atmospheric pressure has been chosen. A fluid model is used. For the electromagnetic part, the three "classical" formulations presented above (MA, PV and B\&S) and the "mixed approach" are used to estimate the self-induced magnetic field. A comparison is made on the results obtained with a current intensity value equal to $600 \mathrm{~A}$ and we conclude on the importance of correctly taking into account the formulation for the selfinduced magnetic field description.

In the first section, we present the model and we detail various formulations to estimate the self-induced magnetic field and particularly the mixed solution. The first results obtained for an academic case corresponding to a cylinder crossed by a constant current intensity will be presented to validate our approach. Then the methods are applied to two geometries to test the method validity. The limits of the method are given in the conclusion.

\section{II/ The model}

\section{$\underline{1 / \text { Fluid model }}$}

In order to describe the arc, we used a stationary fluid model. The Navier - Stokes equation is solved coupled with the mass and energy conservation equations. All these equations are written in the form of the generalised conservation equation (1) where $\phi$ is the variable solved, $\rho$ the mass density, $\vec{v}$ the velocity vector, $\Gamma_{\phi}$ the diffusion coefficient and $S_{\phi}$ the source term. In our case, we consider this equation in a $2 \mathrm{D}$ axisymetrical stationary configuration. All the equations solved for the fluid model are summarized in table 1.

$$
\vec{\nabla}(\rho \vec{v} \phi)=\vec{\nabla}\left(\Gamma_{\phi} \vec{\nabla} \phi\right)+S_{\phi}
$$

In table1, $v_{\mathrm{r}}$ and $\mathrm{v}_{\mathrm{z}}$ represent the radial and axial velocity components respectively, $\mathrm{h}$ is the enthalpy, $\kappa$ and $\mu$ the thermal conductivity and the viscosity. $P$ is the pressure, $j_{r}$ and $j_{z}$ the radial and axial current density components, $\sigma$ and $\mathrm{C}_{\mathrm{p}}$ the electrical conductivity and the heat capacity, $\varepsilon_{\mathrm{n}}$ the net emission coefficient. 


\section{$\underline{\text { 2/ Electric and electromagnetic model }}$}

a) Electric potential equation

In order to describe the electrical properties of the arc, we solved the potential scalar equations designated by the variable $\mathrm{V}$ presented in table 2 .

From this potential, the axial and radial current density components $\mathrm{j}_{\mathrm{z}}$ and $\mathrm{j}_{\mathrm{r}}$ (equations (7-8)) are calculated.

$j_{z}=-\sigma \frac{\partial V}{\partial z}$

$j_{r}=-\sigma \frac{\partial V}{\partial r}$

b) Electromagnetic models

In this study, we used four different formulations to estimate the self-induced magnetic field:

- The Maxwell - Ampere equation (MA)

- The Biot \& Savart equation (B\&S)

- The potential vector with null flux boundary conditions (PVNF)

- The potential vector with null value boundary conditions (PVNV)

- The mixed formulation: Potential vector with B\&S boundary conditions (MF)

\section{- Maxwell Ampere equation}

In the eighties and nineties some authors (for example [1-2]) proposed to calculate the self-induced magnetic field by using the equation:

$B_{\theta}=\frac{\mu_{0}}{R} \int_{0}^{R} r j_{z} d r$

In this equation (9), $\mathrm{R}$ is the integrated radius where the axial component of the current density exists, and $\mu_{0}$ the vacuum permittivity. From this equation the azimuthal magnetic field induced by the arc current is calculated.

This equation comes from the Maxwell-Ampere equation: 
Nevertheless, equation (10) is a vectorial equation and must be verified for each component of the magnetic field vector. So, in case of an axisymetrical system, two equations must be satisfied for the azimuthal magnetic field:

$$
\begin{aligned}
& \frac{1}{r} \frac{\partial}{\partial r}\left(r B_{\theta}\right)=\mu_{0} j_{z} \\
& -\frac{\partial B_{\theta}}{\partial z}=\mu_{0} j_{r}
\end{aligned}
$$

Assuming only equation (9) to deduce the azimuthal component ignores equation (12) and considers $\mathrm{B}_{\theta}$ invariant with the axial coordinate. Moreover, this formulation is only suitable in structured 2D cases.

\section{- Biot \& Savart formulation}

It is possible to obtain the magnetic field using the $B \& S$ equation:

$$
\vec{B}=\frac{\mu_{0}}{4 \pi} \cdot \iiint_{\text {Volume }} \frac{\vec{j} \wedge\left(\vec{r}-\vec{r}^{\prime}\right)}{\left|\vec{r}-\vec{r}^{\prime}\right|^{3}} d V
$$

To use this equation integration over the whole volume must be done for each point. This formulation is valid in $2 \mathrm{D}$ as well as in $3 \mathrm{D}$ geometries. Some authors have proposed the selfinduced magnetic field be calculated by using this approach [3-5]. Nevertheless, the B\&S formulation uses a large part of the computational time as integration over the volume must be done for each point of the mesh.

\section{- Potential vector formulation with null flux boundary condition or null value boundary condtion}

Nowadays, in order to estimate the magnetic field almost all authors use the potential vector formulation: 
$\vec{B}=\vec{\nabla} \wedge \vec{A}$

Where $\vec{A}$ is the potential vector which must verify equation (15), under the Poisson Gauge condition.

$\vec{\Delta} \cdot \vec{A}=\mu_{0} \vec{j}$

In $2 \mathrm{D}$ axisymetrical configuration this leads to the three following equations:

$$
\begin{aligned}
& \frac{\partial^{2} A_{z}}{\partial z^{2}}=\mu_{0} j_{z} \\
& \frac{1}{r} \frac{\partial}{\partial r}\left(r \frac{\partial A_{r}}{\partial r}\right)=-\mu_{0} j_{r}+\frac{A_{r}}{r} \\
& B_{\theta}=\frac{\partial A_{z}}{\partial r}-\frac{\partial A_{r}}{\partial z}
\end{aligned}
$$

This formulation is very useful as equations (16) and (17) can be written in the general form of equation (1) giving a correct estimation of the self-induced magnetic field, taking into account all the components of the current density. Nevertheless, with this formulation a problem remains concerning the choice of the boundary conditions. Indeed, it is generally considered that the components of potential vector $\vec{A}$ tend to zero at an infinite distance. As infinity cannot be reached in the calculation domain, almost all authors consider a null flux for the boundary conditions of equations (16) and (17) or a null value far from the arc [6-10]. In this study, we will call this approach potential vector formulation with null flux boundary condition (PVNF) and the approach with a null value of the potential vector (PVNV)

\section{- Potential vector formulation with $B$ \& $S$ boundary condition}

Another way to obtain the values of the potential vector components consists in using the B\&S formulation:

$$
\vec{A}=\frac{\mu_{0}}{4 \pi} \iiint_{\text {volume }} \frac{\vec{j}}{\vec{r}-\overrightarrow{r^{\prime}} \mid} d V
$$


Obviously, the resolution of equation (19), like that of equation (13), is time consuming. Nevertheless, it can only be used in boundary conditions, whereas within the computational domain equations (16-18) should be used to obtain the self-induced magnetic field. We call this the mixed formulation (MF)

The development of equation (15) in axisymetrical cases requires the use of elliptical integrals. The mathematical approach used is presented in annex A.

\section{III/ Validation of the electromagnetic models}

To validate the magnetic field formulations presented above, we defined a theoretical case consisting of, a constant current density, crossing a cylinder as presented in figure 1 . The cylinder has a radius of $2 \mathrm{~mm}$ and is $40 \mathrm{~mm}$ long. We assumed that it is crossed by a constant current density of $4.10^{6} \mathrm{~A} / \mathrm{m}^{2}$ resulting to a current of $50 \mathrm{~A}$. The current is uniformly distributed in the central part $(\mathrm{r}<\mathrm{R})$. $\mathrm{R}$ is the radius of the part where the axial current density is not equal to zero.

The length of the cylinder is ten-fold its diameter. For this geometry, it is possible to assume analytical equations for $\mathrm{A}_{\mathrm{z}}$, and $\mathrm{B}_{\theta}$ :

$$
\begin{aligned}
& B_{\theta}(r)=\frac{\mu_{0} j_{z} R^{2}}{2 \mathrm{r}} \quad \text { if } r>R \\
& B_{\theta}(r)=\frac{\mu_{0} j_{z} r}{2} \quad \text { if } r<R \\
& A_{z}(r)=A_{z}(0)-\frac{\mu_{0} j_{z} R^{2}}{2}\left(\frac{1}{2}+\ln \left(\frac{r}{R}\right)\right) \text { if } r>R \\
& A_{z}(r)=A_{z}(0)-\frac{\mu_{0} j_{z} r^{2}}{4} \text { if } r<R
\end{aligned}
$$

These equations correspond to the case of an infinite cylinder but, in the present case, are valid in the center plane of the cylinder (central line in figure 1)

Calculations were made for the PVNF, PVNV and the MF models. A comparison of the results obtained for the three approaches and the theoretical data given by equation (20) for the azimuthal value of the magnetic field are compared in figure 2.

In the cylinder $(\mathrm{r}<\mathrm{R}=2 \mathrm{~mm})$, a good agreement was found between the theoretical result and the magnetic field calculated with the three approaches (MF, PVNV and PVNF). 
Outside the cylinder, where there is no current, the MF and PVNV approaches give a better estimation of the magnetic field than PVNF. This is clearly due to the null flux boundary condition in the PVNF calculation. However, at the same time, this simple case does not consider a current density variation in axial or radial directions and only the axial component was considered. It is known that the distance of the boundary condition from the conducting zone can affect the accuracy of the results [12-13]. This point will be studied further in the geometry involving the electric arc.

\section{IV/ Study of PVNF and MF approaches in thermal plasmas}

\section{$\underline{1 / \text { Geometries studied }}$}

In order to study the influence of various magnetic field formulations on the arc behaviour, we compared two academic configurations, presented in figure 3 . In both cases we consider a transferred arc.

The first geometry (Geom1), consists of a cathode (AA'BB') (radius 2mm , length $5 \mathrm{~mm})$ and an anode (YY'ZZ') $(3.5 \mathrm{~mm} ; 4 \mathrm{~mm})$. Near the cathode, an inlet (BC) is assumed. Segment (CDW) is a wall located $15 \mathrm{~mm}$ from the axis. Segment (WXY) is a pressure outlet enabling plasma flow out of the domain. This first geometry was chosen large enough for the arc to be able to expand freely within the volume. It is intended to be representative of a kind of free burning arc.

The second geometry Geom 2 was constructed to be representative of cases where the plasma is more constricted (like in plasma torches or circuit breaker configurations). Moreover, with this geometry we can observe the influence of the distance between the arc fringes and the radial boundary conditions. Also, we can study if ignoring the radial current density component in some formulations leads to a poor estimation of the self-induced magnetic field. Geom2 has the same electrode dimensions as Geom1. The inlet dimension (BC) is unchanged. Segment (CDEFG) is a wall that constitutes a convergent - divergent nozzle. Lines $1 \& 2$ are radial segments where quantities such as the temperature, the velocity or the magnetic field will be presented.

The choice of these two geometries was made to evaluate the influence of the magnetic field calculation for different thermal plasma configurations. 
In both Geom1 and Geom2, air plasma at atmospheric pressure is assumed. The current intensity is equal to $600 \mathrm{~A}$ and the mass inlet flow (BC boundary) is $25 \mathrm{Nl} / \mathrm{min}$. The boundary conditions, except for the potential vectors, are given in table 3.

Concerning the electrical potential, a parabolic profile is imposed on the cathode tip. This is given by equation (22) where $\mathrm{j}_{\max }$ is the maximum value of the current density (chosen equal to $1.210^{8} \mathrm{~A} / \mathrm{m}^{2}$ ) and $\mathrm{R}_{\text {cat }}$ the radius verifying equation (24) for current intensity $\mathrm{I}$. The radial component of the current density is assumed equal to zero.

$$
\begin{array}{ll}
j_{z}(r)=j_{\max }\left(1-\frac{r}{R_{c a t}}\right)^{2} & \text { if } \mathrm{r}<\mathrm{R}_{\text {cat }} \\
j_{z}(r)=0 & \text { if } \mathrm{r}>\mathrm{R}_{\text {cat }} \\
I=2 \pi \int_{0}^{R_{\text {cat }}} j_{z} r d r &
\end{array}
$$

\section{3/ Boundary conditions for the magnetic field}

In order to compare the various formulations, we considered several cases, described in table 4 for Geom 1 and table 5 for Geom2. The generic number of the cases $n^{\circ}$ "X-Y" where $\mathrm{X}$ corresponds to the number of the geometry and $\mathrm{Y}$ the number of the case. All the cases and formulations are presented above and summarized in table 6.

- Cases 1-1, 1-2, 1-3: In some cases, we applied the mixed formulation by taking into account the current density distribution only within the plasma. Nevertheless, current densities also exist in the electrodes and particularly in the cathode. This current in the cathode plays a nonnegligible role on the potential vector amplitude and thus on the magnetic field. In order to study the influence of the cathode, we tested three cases. In case 1-2 only the current density distribution within the plasma is taken into account. In case 1-1 a constant distribution of the axial current density is imposed within the cathode. In the last case (case 1-3) a null flux of the potential vector components near the electrodes is assumed. For case 1-1, a constant distribution for $\mathrm{j}_{\mathrm{z}}$ within the cathode is used. Indeed, Gonzalez [14] have shown that the profile of the current density in the cathode is almost constant and that the change of this constant profile to a more complex one at the boundary between the electrode and the plasma occurs only in a very thin zone. 
- Cases 1-4, 1-5: Case 1-4 is devoted to the results of the PVNF formulation applied to Geom1. This case corresponds to the way this method is usually used in the literature. Nevertheless, to complete and compare the results with case 1-1, we have studied the PVNF formulation taking into account the current density within the cathode (like for case 1-1). This corresponds to case $1-5$.

- Case 1-6 is devoted to the test of the PVNV formulation. Nervetheless, due to the results of cases 1-4 and 1-5, only the case taking into account the current density in the cathode is presented.

- Case 1-7 concerns the situation using the MA formulation in Geom1.

- Cases 2-1, 2-2, 2-3, and 2-4 are a repetition of cases 1-1, 1-4, 1-5 and 1-7 for the constricted geometry Geom2.

- Cases 2-5, 2-6, 2-7 and 2-8: As Geom2 is constricted, we have also tested to impose the boundary conditions for the potential vector in a "radially" extended domain. Thus, for case 2-5 the domain is extended up to the radial value of segment (WX) (that is to say $15 \mathrm{~mm}$ ) and PVNF is applied. Case 2-6 corresponds to the same extended domain but with PVNV formulation. Finally, cases 2-7 and 2-8 are PVNF calculations for an extended radial domain up to respectively $9 \mathrm{~mm}$ and $6 \mathrm{~mm}$.

\section{4/ Results for Geom1.}

\section{a) General results for Geom1}

First, we present "general" results obtained with geometry Geom1. As shown below, the more correct results are obtained by case 1-1. So temperature fields, velocity and current density components are proposed for this case in figures $4 \& 5$ in order to present the general properties of the arc created. In the right hand of figure 4 , it is seen that a temperature maximum of $31 \mathrm{kK}$ is reached on the axis near the cathode. On the axis, the temperature decreases from this maximum to $12 \mathrm{kK}$ near the anode. Along the radial direction the arc expands as expected for this kind of geometry. Concerning the velocity a maximum of $1500 \mathrm{~m} / \mathrm{s}$ is reached under the cathode. In this area, the velocity is essentially created by the Lorentz force produced by the interaction of the self-induced magnetic field and the current densities of the plasma. The velocity decreases along the axis. 
The components of the current density in the geometry are presented in figure 5. The axial component $j_{z}$ is maximal on the cathode where the parabolic condition (22) is imposed. As it exists an expansion of the arc, there is an increase of the conductive section along the axis and thus a decrease of the magnitude of the axial current density. Within the cathode, the constant axial current density that we assumed can be observed. Concerning the radial component of the current density, it is maximal near the cathode with a value of around $-710^{7}$ $\mathrm{A} / \mathrm{m}^{2}$. This maximum is out of the axis. The magnitude of the radial current density decreases away from the cathode.

\section{b) Influence of the magnetic field formulation in Geom1}

In this section, we compare the magnetic field obtained for the different cases (Table 4). The magnetic field obtained in case 1-1 is presented in figure 6 . We can see that it is maximal on the side of the cathode with a value of around $-70 \mathrm{mT}$. This magnetic field is compared on the right part of figure 6 to the one obtained by a numerical calculation of equation (13) for all points of the geometry. We can conclude to a good agreement between the two cases. The magnetic field representation found for case 1-1 is similar to that deduced from the B\&S formulation. Due to the good agreement, the other case will be compared with case 1-1. As the B\&S formulation is the exact one, the comparison validates the MF method taking the current circulation in the cathode into account.

In figure 7, a comparison is given between case 1-1 (MF where the current in the cathode is considered) and case 1-2 (MF where the current in the cathode is not considered). The aim now was to study if the current circulation on the cathode really needs to be considered as this point is never presented in the literature. Dashed lines correspond to levels A to $\mathrm{K}$ for case 1-1 (Left hand side). These lines are reported on the right hand side, related to case 1-2. The dashed lines must therefore be compared to the continuous lines. All figures involving a magnetic field use a similar representation. The magnitude of the magnetic field obtained in case 1-2 in the vicinity of the upper electrode is clearly smaller than in case 1-1, with a value near the cathode equal to $-60 \mathrm{mT}$. This will lead to differences on the estimated convective Lorentz forces. Axially, even if the behaviour of the magnetic field obtained in the two cases is similar, the values obtained without taking the current in the cathode into account remain smaller than those obtained in case 1-1. From this point, we can therefore conclude that the authors who consider the MF (like He Ping Li [11]) or B\&S formulation without considering the current in the cathode, underestimate the magnetic field. 
In figure 8 , we compare case 1-1 and case 1-3, where null flux is considered for potential vector components on the arc attachment locations. In case 1-3, the magnetic field obtained near the cathode is more than $80 \mathrm{mT}$ and so higher than in case 1-1. Nevertheless, in other parts of the calculation domain, the differences between the two fields are weak.

In Figure 9 we plot the magnetic field obtained considering potential vectors with null flux conditions applied at all the boundaries (case 1-4). In this case, neither the radial nor the axial expansions seem to be well predicted. Furthermore, the null flux boundary condition on the potential vector leads to an overestimation of the magnetic field close to the cathode. This case corresponds to the most classical boundary conditions used in the literature when authors use PVNF formulation [9-10].

In order to compare with the MF case where current density is taken into the cathode we achieved a calculation of PVNF with this condition (case 1-5). The magnetic field obtained is plotted in figure 10. We can observe that the magnetic field obtained for PVNF taking into account the cathode is in quite good agreement with MF results on the centre of the plasma. Nevertheless, in the radial direction, the differences between the two formulations remain important.

In figure 11, comparison between case 1-1 and case 1-6 (PVNV taking into account the cathode) is presented. We can observe that a better agreement is obtained for this case even if PVNV condition seems to overestimate the radial values of the magnetic field.

Finally, in figure 12 we plot the magnetic field obtained by the MA equation (ignoring equation (12), case 1-7) and MF (case 1-1). The magnetic field obtained by the MA formulation is higher in the axial and radial directions than that obtained with the MF formulation. The results close to the cathode are suitable compared to those obtained in other parts of the domain such as in the vicinity of the anode.

In order to estimate the influence of these differences on arc properties, axial velocity and temperature profiles obtained in various cases are plotted in figures 13-14-15. In figure 13), we can observe that the behaviour of the velocity profiles following the different formulations is similar with a maximum value located at the position $z=12 \mathrm{~mm}$. This maximum decreases from $12 \mathrm{~mm}$ to the anode position. Cases 1-3, 1-4 and 1-7 lead to similar profile evolutions with a maximum value of $1500 \mathrm{~m} / \mathrm{s}$ at $\mathrm{z}=12 \mathrm{~mm}$. For these three cases, the velocity is overestimated compared to case 1-1 which corresponds to the MF taking into account the cathode. In cases 1-1, 1-5 and 1-6 the maximum velocity value is $1350 \mathrm{~m} / \mathrm{s}$. For these three cases, the current density is taken within the cathode and the formulation used 
seems not to play a role on the evolution of the velocity on the axis. This was not the case for magnetic field values.

Finally, due to the underestimation of the magnetic field in case 1-2, the velocity is clearly underestimated too.

The axial temperature variations following the choice of the different formulations used to represent the magnetic fields are reported in figure 13b. Except for case 1-2, the temperature profiles obtained for the various cases is almost the same. The most important difference of $700 \mathrm{~K}$ between the temperature profiles is obtained between case 1-1 and the others at $\mathrm{z}=15 \mathrm{~mm}$.

We present (Figures 14a\&b and Figures 15a\&b) the radial evolutions for the axial velocity and the temperature for the two sections (line 1 and line 2) represented in the geometries of Figure 3.The velocity profiles are presented for the two lines. The conclusions are the same for the two lines: in cases 1-3, 1-4, 1-7 an overestimation of the axial velocity is found compared to the ones obtained in cases 1-1, 1-5 and 1-6. The velocity obtained in case 1-2 is underestimated.

The greatest difference occurs close to the axis for plasma radii lower than the electrode. This difference becomes lower going to the anode position.

For a temperature representation, the axial and radial temperature evolutions (Figure $13 \mathrm{~b}$ and also Figures $15 \mathrm{a} \& \mathrm{~b}$ ) shows that MA, PVNF, MF, PVNV with or without current circulation in the cathode are sufficient to represent the temperature field correctly.

In figures $15 \mathrm{a} \& \mathrm{~b}$, we present the radial temperature profiles for line 1 and line 2 . The differences in the approaches used to calculate the self-induced magnetic fields are very small for temperature variation.

\section{5/ Results for geometry 2.}

In this section, we studied if the conclusions obtained with Geom1 remain the same for a geometry where the arc is constricted (Geom2). All the cases studied are summarized in table 5. First, the general results obtained in case 2-1 (M.F. With cathode) are presented. Secondly, the influence of the approach used to calculate the self-induced magnetic field is commented. For the second step, only PVNF, MA and MF are studied due to the fact that boundary conditions are too close to the conduction core to apply PVNV. Finally, a study on the position of an extended radial boundary condition for PVNF and PVNV is proposed.

\section{a) General results for Geom 2}


The temperature and velocity fields obtained in case 2-1 are presented in figure 16. For the velocity (left hand side in figure 16), the nozzle presence accelerates the fluid in order to maintain the mass flow rate. The maximum plasma velocity is $2000 \mathrm{~m} / \mathrm{s}$ and is reached on the axis at the nozzle exit. The maximum temperature (around $31 \mathrm{kK}$ ) is obtained close to the cathode tip. In the nozzle, the temperature decreases to $16 \mathrm{kK}$ at the nozzle exit.

Figure 17, the axial current density is maximum near the cathode where equation (22) is assumed as boundary condition. Along the axis, current density decreases as conduction radius increases. Concerning the radial current density, values up to $10^{6} \mathrm{~A} / \mathrm{m}^{2}$ are obtained near the cathode and at the exit of the throat of the nozzle.

\section{b) Influence of magnetic field formulation}

In this section, the influence of the magnetic field formulation is studied in geometry 2. As shown in the study with Geom1, the case where MF is used without taking into account the electrode shows a clear underestimation of the velocity. Consequently, MF formulation without the cathode is no longer considered.

Magnetic fields obtained in case 2-2 (PVNF), case 2-3 (PVNF with cathode) and in case 2-4 (MA) are compared with the case 2-1 (MF with cathode) (Figures 18,19,20)

It seems clear that the estimation given by the PVNF formulation (Cases 2-2, 2-3) is far from the reference solution (figures 18,19). These differences are present in the axial and radial directions. Furthermore, in the nozzle, the null flux boundary condition leads to a quasi null value of the magnetic field which is not physical. The trend obtained in the previous geometry with the comparison of formulations MA and MF with cathode is conserved (figure 20). Thus, MA leads to an overestimation of the magnetic field in the radial direction and to a lesser extension, in the axial direction.

As with Geom1 we present the influence of the magnetic field formulation on the velocity and temperature profiles which are plotted along the axis in figures $21 \mathrm{a} \& \mathrm{~b}$. The axial velocity profile on the axis is different for the four cases. In all geometries, MA and PVNF without taking into account the current within the cathode lead to an overestimation of the velocity compared with MF. The greatest difference $(350 \mathrm{~m} / \mathrm{s})$ is obtained at $\mathrm{z}=12.5 \mathrm{~mm}$. Note that differences also exist between MA and PVNF, particularly in the throat of the nozzle and after the exit $(\mathrm{z}=25 \mathrm{~mm})$. For case 2-3 which corresponds to the PVNF where current in the cathode has been taken into account, before the nozzle throat, the values of the velocity are close to the one of the MF case. Nevertheless, after the throat, the velocity is clearly underestimated, probably due to the bad estimation of the magnetic field. 
Whereas velocities on the axis are different, the axial temperature profile does not seem to depend on the formulation used for the magnetic field.

c) Influence of the domain dimension for the boundary condition for PVNF..

It seems that PVNF work "well" when null flux is applied to a boundary far from the conduction zone. In this section, we achieve a calculation, in Geom2 but with the potential vector equation solved on an extended domain. All cases calculated are presented in table 5 .

Case 2-5 calculates the potential vectors beyond the nozzle wall in an extended domain up to a radial boundary situated at $r=15 \mathrm{~mm}$ (in front of line WX (figure 3)).

- $\quad$ Case 2-6 corresponds to the same extended domain but applying PVNV.

- $\quad$ Cases 2-7 and 2-8 are calculations for respectively $9 \mathrm{~mm}$ and $6 \mathrm{~mm}$ extended domain.

The dimensions $15 \mathrm{~mm}, 9 \mathrm{~mm}$ and $6 \mathrm{~mm}$ have been chosen as they corresponds approximately to respectively 5, 3 and 2 times the characteristic arc core radius. The new calculation domain for Geom2 is presented in figure 22. Dashed domain corresponds to the extended domain for potential vector calculation.

The comparison of the magnetic field obtained with the mixed formulation and PVNF with full extended domain taking into account the cathode is presented in figure 23 . In the plasma, the estimation of the magnetic field obtained with the extended domain is clearly closer to the reference than the one obtained without the extended. Nevertheless, as obtained for Geom1, the radial evolution far from the plasma (visible close to the anode) is badly represented.

In order to study the influence of the dimension of the extended domain, we propose to study the evolution of the magnetic field along the isotherm $10 \mathrm{kK}$ in figure 24 . This isotherm can be seen as an image of the conduction zone and its position remains the same for all cases studied in Geom2. The evolution of magnetic field is plotted for a radial extended domain of $9 \mathrm{~mm}$ (case 2-6) and 6mm (case 2-7). To complete the study, the magnetic field obtained with PVNV is also presented on the same figure. The results obtained with PVNV formulation are very close to those of the mixed formulation. For the PVNF formulation, results from $15 \mathrm{~mm}$ and $9 \mathrm{~mm}$ extended domains are also close to the reference case with slight differences. When the size of the extended domain is too reduced compared to the conduction zone (case 2-7 with $6 \mathrm{~mm}$ extended domain), one can observe that the estimation of the magnetic field is clearly worse. 
In order to conclude on the influence of the size of the extended domain on the arc characteristics, the evolution of the axial velocity for the various cases is represented in figure 25. For PVNF used with $15 \mathrm{~mm}$ and $9 \mathrm{~mm}$ extended domain and for PVNV case, the profile of the axial velocity obtained is close to the reference one but shifted by around $50 \mathrm{~m} / \mathrm{s}$. As expected, due to differences observed on the magnetic fields calculated for the case 2-7 the velocity profile is further away from the reference one. Nevertheless, the maximum difference remains under $200 \mathrm{~m} / \mathrm{s}$.

\section{V/ Conclusion}

The self-induced magnetic field conjugated with the current density leads to the Lorentz forces. These forces generate velocity in the arc core and, depending on the cathode geometry and the injected mass flow rate, can be totally responsible for the plasma characteristics. The self-induced magnetic field is due to current circulation not only in the plasma but also in the electrodes. Nevertheless current circulation in the electrode is never or rarely taken into account except in low-voltage circuit-breaker studies to represent arc displacement [15]. Several methods exist to represent the self-induced magnetic field such as the Biot \& Savart formulation (which is the exact solution but is very time consuming), the Maxwell Ampere formulation (in two dimensional systems), the vector potential formulation (VP) with a null flux(PVNF) or an null value (PVNV) boundary condition on the walls. Due to the lack of studies on this point, this article was devoted to the comparison of several methods in two different geometries (Geom1 and Geom2). As the difficulty of the vector potential formulation is the choice of the boundary conditions, we propose a mixed formulation (MF) where the boundary values of the vector potential are determined by the resolution of the Biot \& Savart formulation written for the vector potential. As the aim was also to obtain a satisfactory method in $2 \mathrm{D}$, the elliptic integrals are used to assume the boundary conditions. Geom2 was a confined geometry compared to Geom1, with the applied boundary conditions close to the arc core. A study is also proposed on the interest of calculating the potential vector on an extended domain in such case. Table 6 summarize the results of the study which allow conclusions to be reached on several points:

- The self-induced magnetic field produced by current circulation in the cathode is essential to represent the velocity field. For PVNF and PVNV formulations, only this approach provides results comparable with the Biot \& Savart formulation. 
- For a representation of the temperature field, other methods such as PVNF (Vector potential with a null flux) or MA (Maxwell Ampere) give similar results.

- When the wall boundary condition of the geometry is close to the arc core, the PVNF formulation must be used with an extended domain on the radial direction for the potential vector calculation. The extension of this domain should be up to 3 times the characteristic arc core radius.

- The mixed formulation provides exactly the same results as the Biot \& Savart formulation but requires much less calculation time. Indeed the triple integral is only calculated for points along the domain edges.

- The mixed formulation gives a good representation of the velocity field in the calculation domain. Indeed even though small differences exist in the temperature field, a wrong estimation of the self-induced magnetic field leads to large differences in the deduced velocities.

- The present study used a current intensity of 600A, in the stationary case. An increase of the current intensity leads to an increase of the self-induced magnetic field and can give erroneous results for the velocity field and the pressure waves in specific configurations such as for example high-voltage circuit breakers where the current intensity can reach $70 \mathrm{kA}$. 


\section{Appendix A}

$\underline{\text { Boundary condition for } \mathrm{A}_{\underline{z}}}$ (A1).

In order to calculate the Biot \& Savart condition for $\mathrm{A}_{\mathrm{z}}$ we have to remember equation

$$
\frac{A_{z}(\vec{r})}{\mu_{0}}=\frac{1}{4 \Pi} \iiint_{V} \frac{j_{z}\left(\overrightarrow{r^{\prime}}\right)}{\left|\vec{r}-\overrightarrow{r^{\prime}}\right|} d V
$$

In order to estimate the term $\left|\vec{r}-\overrightarrow{r^{\prime}}\right|$ in cylindrical axis we propose to use figure A1

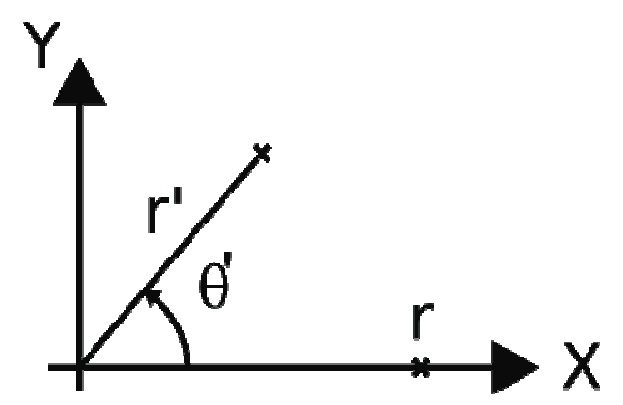

Figure A1

So we have in Cartesian $\vec{r}\left(\begin{array}{l}x \\ y \\ z\end{array}\right)$ and $\vec{r}\left(\begin{array}{l}x^{\prime} \\ y^{\prime} \\ z^{\prime}\end{array}\right)$ and so in cylindrical formulation $\vec{r}\left(\begin{array}{l}r \\ 0 \\ z\end{array}\right)$ and $\vec{r}\left(\begin{array}{c}r^{\prime} \cdot \cos \left(\theta^{\prime}\right) \\ r^{\prime} \cdot \sin \left(\theta^{\prime}\right) \\ z^{\prime}\end{array}\right)$. So equation (A1) can be rewritten in term of cylindrical data.

$$
\frac{A_{z}(r, z)}{\mu_{0}}=\frac{1}{4 \Pi} \iiint_{0,-\Pi, z_{1}} \frac{j_{z}\left(r^{\prime}, z^{\prime}\right)}{\left[\left(r-r^{\prime} \cos \theta^{\prime}\right)^{2}+\left(r^{\prime} \sin \theta^{\prime}\right)^{2}+\left(z-z^{\prime}\right)^{2}\right]^{(1 / 2)}} r^{\prime} d r^{\prime} d \theta \theta d z^{\prime}
$$

Assuming that $\mathrm{j}_{\mathrm{z}}$ is constant for $\theta$, we have 


$$
\frac{A_{z}(r, z)}{\mu_{0}}=\frac{1}{4 \Pi} \iint_{0, z_{1}} j_{z}\left(r^{\prime}, z^{\prime}\right) r^{\prime}\left[\int_{-\Pi}^{\Pi} \frac{1}{\left[\left(r-r^{\prime} \cos \theta^{\prime}\right)^{2}+\left(r^{\prime} \sin \theta^{\prime}\right)^{2}+\left(z-z^{\prime}\right)^{2}\right]^{(1 / 2)}} d \theta^{\prime}\right] d r^{\prime} d z^{\prime}
$$

If we consider elliptical integral $I_{(0, n, n)}\left(r, r^{\prime}, a\right)$ defined by equation (A4), the integral over $\theta$ can be written in function of $I_{(0,0,0)}\left(r, r^{\prime}, a\right)$ written in equation (A5) in function of the complete first order elliptical integral $\mathrm{K}(\mathrm{k})$.

$$
\begin{gathered}
I_{(0, n, n)}\left(r, r^{\prime}, a\right)=\frac{1}{\Pi} \int_{0}^{\Pi} \frac{\cos (n \theta)}{\left(r^{2}+r^{\prime 2}+a^{2}-2 r r^{\prime} \cos \theta\right)^{(1 / 2)}} \cdot d \theta \\
I_{(0,0,0)}\left(r, r^{\prime}, a\right)=\frac{k K(k)}{\Pi \sqrt{\left(r r^{\prime}\right)}}
\end{gathered}
$$

With :

$$
k=\left[\frac{4 r r^{\prime}}{\left(r+r^{\prime}\right)^{2}+z^{2}}\right]^{(1 / 2)}
$$

And

$$
K(k)=\int_{0}^{\Pi / 2} \frac{d \theta}{1-k^{2} \sin ^{2}(\theta)}
$$

More details can be found in [16] and [17]. From equation (A4) we can rewrite equation (A2) in term of elliptical integral :

$$
\frac{A_{z}(r, z)}{\mu_{0}}=\frac{1}{2} \iint_{0, z_{1}} j_{z}\left(r^{\prime}, z^{\prime}\right) r^{\prime} I_{(0,0,0)}\left(r, r^{\prime}, z-z^{\prime}\right) d r^{\prime} d z^{\prime}
$$

The strong interest of equation (A7) is that $\mathrm{I}_{(0,0,0)}$ can be expressed in terms of $\mathrm{K}(\mathrm{k})$ and that $\mathrm{K}(\mathrm{k})$ can be approximated by an analytical equation :

$$
\begin{aligned}
& K(k)=\left(a_{0}+a_{1} m_{1}+a_{2} m_{1}^{2}+a_{3} m_{1}^{3}+a_{4} m_{1}^{4}\right) \\
& +\ln \left(1 / m_{1}\right)\left(b_{0}+b_{1} m_{1}+b_{2} m_{1}^{2}+b_{3} m_{1}^{3}+b_{4} m_{1}^{4}\right)
\end{aligned}
$$

With $\mathrm{m}_{1}=1-\mathrm{k}^{2}, \mathrm{a}_{\mathrm{i}}$ and $\mathrm{b}_{\mathrm{i}}$ are constants given in [16].

\section{$\underline{\text { Boundary condition for } \mathrm{A}_{r}}$}


For calculating boundary condition for $A_{r}$, we have to use equation (A10).

$$
\frac{A_{r}(\vec{r})}{\mu_{0}}=\frac{1}{4 \Pi} \iiint_{V} \frac{j_{r}\left(\overrightarrow{r^{\prime}}\right)}{\left|\vec{r}-\vec{r}^{\prime}\right|} d V
$$

This equation can be written in cylindrical system :

$$
\frac{A_{r}(r, z)}{\mu_{0}}=\frac{1}{4 \Pi} \iint_{z_{1}, 0} r^{\prime} j_{z}\left(r^{\prime}, z^{\prime}\right) \int_{-\Pi}^{\Pi} \frac{\cos (\theta) d \theta}{\left[r^{2}+r^{\prime 2}+\left(z-z^{\prime}\right)^{2}-2 \mathrm{rr}^{\prime} \cos (\theta)\right]^{(1 / 2)}} d r^{\prime} d z^{\prime}
$$

Equation (A11) can be written in terms of elliptical integrals $I_{(0,1,1)}$ :

$$
\frac{A_{r}(r, z)}{\mu_{0}}=\frac{1}{2} \iint_{z_{1}, 0} r^{\prime} j_{z}\left(r^{\prime}, z^{\prime}\right) I_{(0,1,1)}\left(r, r^{\prime}, z-z^{\prime}\right) d r^{\prime} d z^{\prime}
$$

The integral $\mathrm{I}_{(0,1,1)}$ can be written in term of second and first order total elliptic integrals $\mathrm{K}(\mathrm{k})$ and $\mathrm{E}(\mathrm{k})$

$$
I_{(0,1,1)}=\frac{1}{\Pi k \sqrt{\left(r r^{\prime}\right)}}\left(\left(2-k^{2}\right) K(k)-2 E(k)\right)
$$

As $\mathrm{K}(\mathrm{k})$ in equation (A9), $\mathrm{E}(\mathrm{k})$ can be approximated by an analytical expression [16]

$$
\begin{aligned}
& E(k)=\left(a_{0}+a_{1} m_{1}+a_{2} m_{1}^{2}+a_{3} m_{1}^{3}+a_{4} m_{1}^{4}\right)+ \\
& \ln \left(1 / m_{1}\right)\left(b_{0}+b_{1} m_{1}+b_{2} m_{1}^{2}+b_{3} m_{1}^{3}+b_{4} m_{1}^{4}\right)
\end{aligned}
$$

Values for the coefficients for the calculation of $\mathrm{E}(\mathrm{k})$ and $\mathrm{K}(\mathrm{k})$ are given in table A1.

\begin{tabular}{|c|c|c|c|c|c|}
\hline & $\mathrm{a} 0$ & $\mathrm{a} 1$ & $\mathrm{a} 2$ & $\mathrm{a} 3$ & $\mathrm{a} 4$ \\
\hline $\mathrm{K}(\mathrm{k})$ & 1.38629436112 & 0.09666344259 & 0.03590092383 & 0.03742563713 & 0.01451196212 \\
\hline $\mathrm{E}(\mathrm{k})$ & 0 & 0.44325141463 & 0.06260601220 & 0.04757383546 & 0.01736506451 \\
\hline & $\mathrm{b} 0$ & $\mathrm{~b} 1$ & $\mathrm{~b} 2$ & $\mathrm{~b} 3$ & $\mathrm{~b} 4$ \\
\hline $\mathrm{K}(\mathrm{k})$ & 0.5 & 0.12498593597 & 0.06880248576 & 0.03328355346 & 0.00441787012 \\
\hline $\mathrm{E}(\mathrm{k})$ & 0 & 0.24998368310 & 0.09200180037 & 0.03328355346 & 0.00526449639 \\
\hline
\end{tabular}

Table A1 : Numerical constants for the calculation of the elliptic integrals. 


\section{Table captions}

Table 1: Equations solved in the fluid model

Table 2: Vector potential equation

Table 3: Boundary conditions for geometries Geom1 \& Geom2

Table 4: Description of the magnetic field treatment for the cases studied for Geom1

Table 5: Description of the magnetic field treatment for the cases studied for Geom2

Table 6: Summary of the studied cases and of the results on the estimation of the magnetic field (:) : Good, $: \cdot$ : Average ,: :Bad) 


\begin{tabular}{|c|c|c|c|c|}
\hline Name & $\phi$ & $\Gamma_{\phi}$ & $\mathrm{S}_{\phi}$ & \\
\hline Mass conservation & 1 & 0 & 0 & (2) \\
\hline Axial momentum & $\mathrm{v}_{\mathrm{z}}$ & $\mu$ & $\begin{array}{l}-\frac{\partial p}{\partial z}+\frac{1}{r} \frac{\partial}{\partial r}\left(r \mu \frac{\partial v_{r}}{\partial z}\right)+\frac{1}{3} \frac{\partial}{\partial z}\left(\mu \frac{\partial v_{z}}{\partial z}\right) \\
-\frac{2}{3} \frac{\partial}{\partial z}\left(\frac{1}{r} \frac{\partial}{\partial r}\left(r v_{r}\right)\right)+j_{r} B_{\vartheta}\end{array}$ & (3) \\
\hline Radial momentum & $\mathrm{V}_{\mathrm{r}}$ & $\mu$ & $\begin{array}{l}-\frac{\partial p}{\partial r}+\frac{1}{r} \frac{\partial}{\partial r}\left(r \mu \frac{\partial v_{r}}{\partial z}\right)+\frac{1}{3} \frac{\partial}{\partial z}\left(\mu \frac{\partial v_{z}}{\partial z}\right) \\
-\frac{2}{3} \frac{\partial}{\partial z}\left(\frac{1}{r} \frac{\partial}{\partial r}\left(r v_{r}\right)\right)-j_{z} B_{\vartheta}\end{array}$ & (4) \\
\hline Energy & $\mathrm{h}$ & $\frac{\kappa}{C_{p}}$ & $v_{z} \frac{\partial p}{\partial z}+v_{r} \frac{\partial p}{\partial r}+\mu\left[\begin{array}{l}2\left[\left(\frac{\partial v_{r}}{\partial r}\right)^{2}+\frac{v_{r}^{2}}{r^{2}}+\left(\frac{\partial v_{z}}{\partial z}\right)\right. \\
+\left[\frac{\partial v_{r}}{\partial z}+\frac{\partial v_{z}}{\partial r}\right]^{2}+\left[\frac{\partial v_{\theta}}{\partial z}\right] \\
+\left[\frac{\partial v_{\vartheta}}{\partial r}-\frac{v_{\vartheta}}{r}\right]^{2} \\
+\frac{2}{3}\left[\frac{1}{r} \frac{\partial}{\partial r}\left(r v_{r}\right)+\frac{\partial v_{z}}{\partial z}\right]^{2} \\
+\frac{j_{x}^{2}+j_{r}^{2}}{\sigma}-4 \pi \varepsilon_{N}+\frac{5}{2} \frac{k}{e}\left(\frac{j_{z}}{C_{p}} \frac{\partial h}{\partial z}+\frac{j_{r}}{C_{p}} \frac{\partial h}{\partial r}\right)\end{array}\right.$ & (5) \\
\hline
\end{tabular}

Table 1: Equations solved in the fluid model 


\begin{tabular}{|c|c|c|c|c|}
\hline Name & $\phi$ & $\Gamma_{\phi}$ & $S_{\phi}$ & \\
\hline Electric potential & $\mathrm{V}$ & $\sigma$ & 0, No convective term & $(6)$ \\
\hline
\end{tabular}

Table 2: Vector potential equation 


\begin{tabular}{|c|c|c|c|c|}
\hline Boundary & $\begin{array}{c}\text { Temperature } \\
\text { (T) }\end{array}$ & $\begin{array}{c}\text { Pressure } \\
\text { (P) }\end{array}$ & $\begin{array}{c}\text { Velocity } \\
\text { components } \\
\left(\mathrm{v}_{\mathrm{i}}\right)\end{array}$ & $\begin{array}{l}\text { Scalar potential } \\
\text { (V) }\end{array}$ \\
\hline$A^{\prime}-B^{\prime}$ & $3000 \mathrm{~K}$ & - & $0 \mathrm{~m} / \mathrm{s}$ & $\begin{array}{c}\text { Current density } \\
\text { profile (22) }\end{array}$ \\
\hline B-B' & $300 \mathrm{~K}$ & - & $0 \mathrm{~m} / \mathrm{s}$ & $\frac{\partial V}{\partial n}=0$ \\
\hline B-C & $300 \mathrm{~K}$ & $25 \mathrm{Nl} / \mathrm{min}$ & $\begin{array}{c}\mathrm{v}_{\mathrm{z}} \\
\mathrm{v}_{\mathrm{r}}=0\end{array}$ & $\frac{\partial V}{\partial n}=0$ \\
\hline $\begin{array}{c}(\text { Geom1) C- } \\
\text { D } \\
\text { (Geom2) C- } \\
\text { D-E-F-G- } \\
\text { H-W }\end{array}$ & $300 \mathrm{~K}$ & - & $0 \mathrm{~m} / \mathrm{s}$ & $\frac{\partial V}{\partial n}=0$ \\
\hline W-X-Y & $300 \mathrm{~K}$ & $1 \mathrm{~atm}$ & $\frac{\partial v_{i}}{\partial n}=0$ & $\frac{\partial V}{\partial n}=0$ \\
\hline$Y-Y^{\prime}$ & $300 \mathrm{~K}$ & - & $0 \mathrm{~m} / \mathrm{s}$ & $\frac{\partial V}{\partial n}=0$ \\
\hline$Y^{\prime}-Z^{\prime}$ & $\frac{\partial T}{\partial n}=0$ & - & $0 \mathrm{~m} / \mathrm{s}$ & $0 \mathrm{~V}$ \\
\hline$A-Z^{\prime}$ & $\frac{\partial T}{\partial n}=0$ & $\frac{\partial P}{\partial n}=0$ & $\frac{\partial v_{i}}{\partial n}=0$ & $\frac{\partial V}{\partial n}=0$ \\
\hline
\end{tabular}

Table 3 : Boundary conditions for geometries $1 \& 2$. 


\begin{tabular}{|c|c|c|}
\hline & Geometry & Magnetic field treatment \\
\hline Case 1-1 & Geom 1 : Freely expansion & $\begin{array}{l}\text { - M.F. } \\
\text { - Biot \& Savart on (A'-B'-B-C-D-W-X-Y-Y'-Z'), } \\
\text { calculated from the current density within the } \\
\text { domain and the cathode }\end{array}$ \\
\hline Case 1-2 & Geom1 & $\begin{array}{l}\text { - M.F. } \\
\text { - Biot \& Savart on (A'-B'-B-C-D-W-X-Y-Y'-Z'), } \\
\text { calculated from the current density within the } \\
\text { domain }\end{array}$ \\
\hline Case 1-3 & Geom1 & $\begin{array}{l}\text { - M.F. } \\
\text { - Biot \& Savart on (B'-B-C-D-W-X-Y-Y'), } \\
\text { calculated from the current density within the } \\
\text { domain } \\
\text { - Null flux of potential vector on }\left(Y^{\prime}-Z^{\prime}\right) \text { and }\left(A^{\prime}-B^{\prime}\right)\end{array}$ \\
\hline Case 1-4 & Geom 1 & $\begin{array}{l}\text { - P.V.N.F., on all boundaries, without taking into } \\
\text { account the cathode }\end{array}$ \\
\hline Case 1-5 & Geom1 & $\begin{array}{l}\text { - P.V.N.F., on all boundaries, calculated from the } \\
\text { current density within the domain and the cathode. }\end{array}$ \\
\hline Case 1-6 & Geom1 & $\begin{array}{l}\text { - P.V.N.V. on (DWX) and P.V.N.F. on (Y'-Z') and } \\
\text { (A-B). Case calculated by taking into account the } \\
\text { current density within the domain and the cathode. }\end{array}$ \\
\hline Case 1-7 & Geom1 & - M.A. \\
\hline
\end{tabular}

Table 4 : Description of the magnetic field treatment for the studied cases for Geom 1. 


\begin{tabular}{|c|c|c|}
\hline Case 2-1 & Geom2 & $\begin{array}{l}\text { - M.F. } \\
\text { - Biot \& Savart on (A'-B'-B-C-D-E-F-G-H-W-X-Y- } \\
\text { Y'-Z'), calculated from the current density within the } \\
\text { domain and the cathode }\end{array}$ \\
\hline Case 2-2 & Geom2 & $\begin{array}{l}\text { - P.V.N.F., on all boundaries, without taking into } \\
\text { account the current density within the cathode }\end{array}$ \\
\hline Case 2-3 & Geom2 & $\begin{array}{l}\text { - P.V.N.F., on all boundaries, taking into account } \\
\text { the current density within the cathode }\end{array}$ \\
\hline Case 2-4 & Geom 2 & - M.A. \\
\hline Case 2-5 & Geom2 & $\begin{array}{l}\text { - P.V.N.F., on all boundaries, taking into account } \\
\text { the current density within the cathode and imposed } \\
\text { at an extended radial distance } r=15 \mathrm{~mm}\end{array}$ \\
\hline Case 2-6 & Geom2 & $\begin{array}{l}\text { - P.V.N.V. taking into account the current density } \\
\text { within the cathode and imposed at an extended } \\
\text { radial distance } r=15 \mathrm{~mm} \text {. P.V.N.F. on (ABC) and } \\
(\mathrm{ZYX})\end{array}$ \\
\hline Case 2-7 & Geom2 & $\begin{array}{l}\text { - P.V.N.F. taking into account the current density } \\
\text { within the cathode and imposed at an extended } \\
\text { radial distance } r=9 \mathrm{~mm} \text { P.V.N.F. on }(\mathrm{ABC}) \text { and } \\
(\mathrm{ZYX})\end{array}$ \\
\hline Case 2-8 & Geom 2 & $\begin{array}{l}\text { - P.V.N.F., on all boundaries, taking into account } \\
\text { the current density within the cathode and imposed } \\
\text { at an extended radial distance } r=6 \mathrm{~mm}\end{array}$ \\
\hline
\end{tabular}

Table 5 : Description of the magnetic field treatment for the studied cases for Geom 2. 


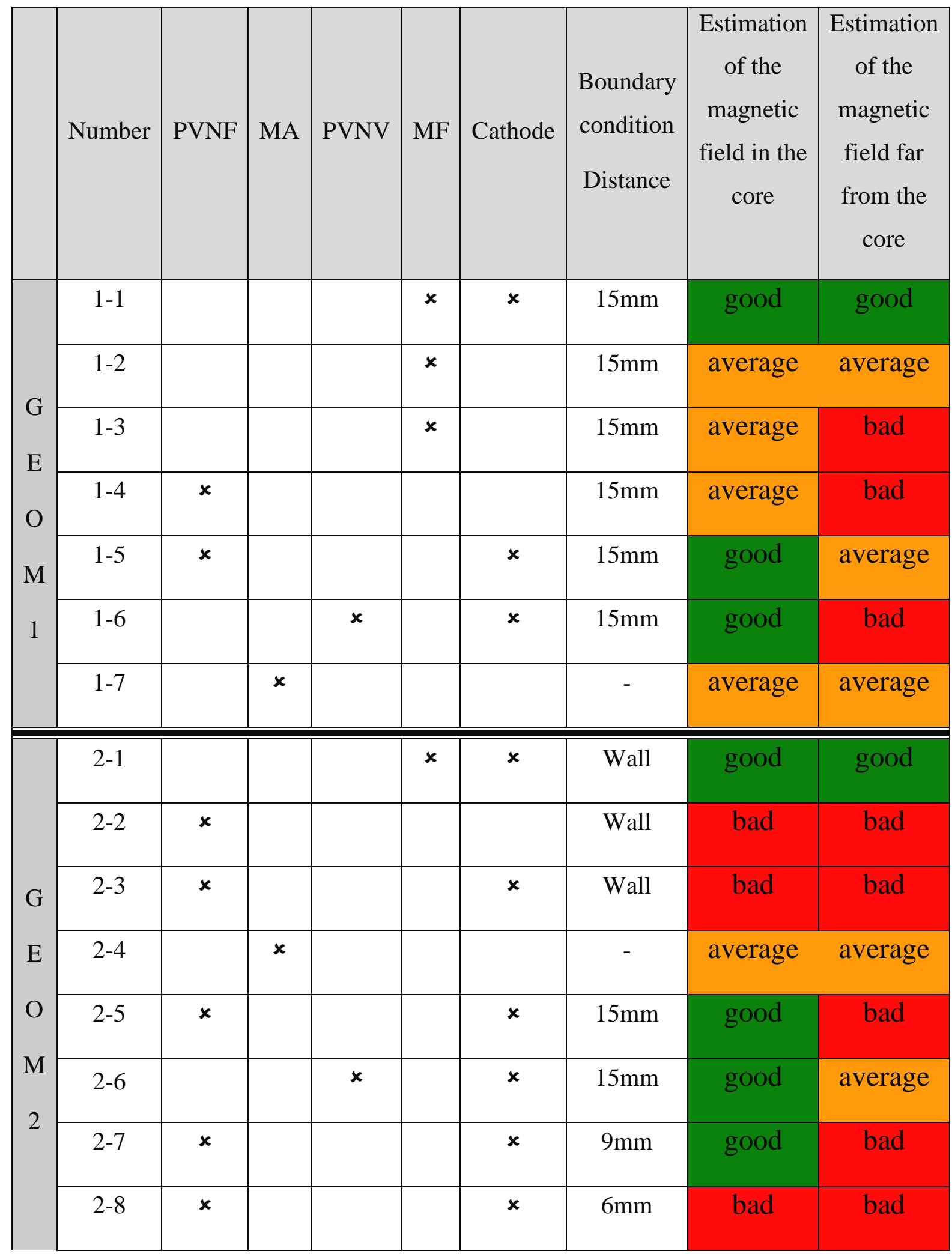

Table 6: Summary of the studied cases and of the results on the estimation of the magnetic field 


\section{$\underline{\text { References }}$}

[1] K.C. Hsu, K. Etemadi and E. Pfrnder, J. Appl. Phys., 54, n³ (1983) p1293.

[2] J J Lowke, R Morrow and J Haidar, J. Phys. D: Appl. Phys., 30, (1997), pp2033-2042

[3] G. Speckhofer, H.-P. Schmidt, IEEE Trans. on Plasma Science 24, 4 (1996) pp12391248

[4] L.Z. Schlitz, S.V. Garimella ans S. H. Chan., Journal of Applied Physics, 85, n5, (1999), pp2540-2546

[5] T. Daube, H. Stammberger, M. Anheuser, and C. Dehning, "3D simulation of a low voltage switching arc based on MHD equations," in Proc. $14^{\text {th }}$ Symp. Phys. Switching Arc (Czech), Sep. 10-14, 2001, pp. 10-14.

[6] D. Bernardi, V. Colombo, E. Ghedini, S. Melini, and A. Mentrelli, IEEE Trans. on plasma science, $\mathrm{v} 33, \mathrm{~N}^{\circ} 2,(2005)$

[7] A Blais, P Proulx and M I Boulos, J. Phys. D: Appl. Phys., 36, (2003), 488-496

[8] P Freton, J J Gonzalez and A Gleizes, J. Phys. D: Appl. Phys., 33, (2000), p2442

[9] J P Trelles, E Pfender and J V R Heberlein, J. Phys. D: Appl. Phys., 40, (2007), 5635

[10] Anthony B Murphy, J. Phys. D: Appl. Phys. 44 (2011), 194009, (11pp)

[11] He-Ping Li and Xi Chen 2001 J. Phys. D: Appl. Phys. 34 L99

[12] D. Bernardi, V. Colomboa, E. Ghedini, and A. Mentrelli, Eur. Phys. J. D, (2003), 27, $55-72$

[13] S. Xue, P. Proulx and M. I. Boulos, J. Phys. D: Appl. Phys., 34, (2001) 1897

[14] J J Gonzalez , F Cayla , P Freton and P Teulet, J. Phys. D: Appl. Phys., 42, (2009), 204

[15] B Swierczynski, J J Gonzalez, P Teulet, P Freton and A Gleizes, J. Phys. D: Appl. Phys., 37, (2004), 595

[16] Abramovitz M. and Stetgum I. S. 1972 Handbook of mathematical functions New York Dover Publications ISBN 0-486-61272-4

[17] J. T. Conway, Transactions on magnetics, 37 (4), 2001), 2977-88 


\section{Figure Captions}

Figure 1: Cylinder with a constant current density distribution

Figure 2: Azimuthal magnetic field in the middle plane of the cylinder

Figure 3: The two geometries studied: (Geom1 ) free expansion geometry, (Geom2) constricted geometry.

Figure 4: Fields of temperature (left) and velocity magnitude (right) for case 1-1

Figure 5: Fields of current density components for case 1-1

Figure 6: Comparison of magnetic field obtained by the mixed formulation (case 1-1) and the full Biot \& Savart Calculation in case of Geom1.

Figure 7: Comparison of azimuthal components of the magnetic field obtained in case 1-1 (M.F. with cathode) and case 1-2 (MF without cathode)

Figure 8: Comparison of azimuthal component of the magnetic field obtained in case 1-1 and case 1-3 (M.F. With null flux on the arc attachment locations: anode and cathode).

Figure 9: Comparison of azimuthal component of the magnetic field obtained in case 1-1 and case 1-4 (P.V.N.F.)

Figure 11: Comparison of azimuthal component of the magnetic field obtained in case 1-1 and case 1-6 (P.V.N.V.)

Figure 12: Comparison of azimuthal component of the magnetic field obtained in case 1-1 and case 1-7 (M.A.) 
Figure 13 a \& b: Axial velocity and temperature in Geom1.

Figure 14 a \& b: Velocity profiles along lines 1 and 2 (Respectively $z=12.5 \mathrm{~mm}$ and $20 \mathrm{~mm}$ )

Figure 15 a \& b: Temperature profiles along lines 1 and $2(z=12.5 \mathrm{~mm}$ and $20 \mathrm{~mm}$ respectively)

Figure 16: Fields of temperature and of the magnitude of the velocity for case 2-1 (MF with cathode)

Figure 17: Current density components

Figure 18: Comparison of azimuthal component of the magnetic field obtained in case 2-1 (MF with cathode) and case 2-2 (PVNF without cathode)

Figure 19: Comparison of azimuthal component of the magnetic field obtained in case 2-1 (M.F. with cathode) and case 2-3 (PVNF with cathode)

Figure 20: Comparison of azimuthal component of the magnetic field obtained in case 2-1 (M.F. with cathode) and case 2-4 (MA)

Figure 21a \& b: Axial velocity (a) and the axial temperature (b) profiles in Geom2.

Figure $22:$ Geom2 with hatched extended domain

Figure 23 : Comparison of azimuthal component of the magnetic field obtained in case 2-1 (M.F. with cathode) and case 2-5 (PVNF extended domain 15mm)

Figure 24: Comparison of the magnetic field along isotherm $10 \mathrm{kK}$, depending on the size of the extended domain and the formula. 
Figure 25: Evolution of the axial velocity in geom. 2 depending on the size of the extended domain and the formulation. 


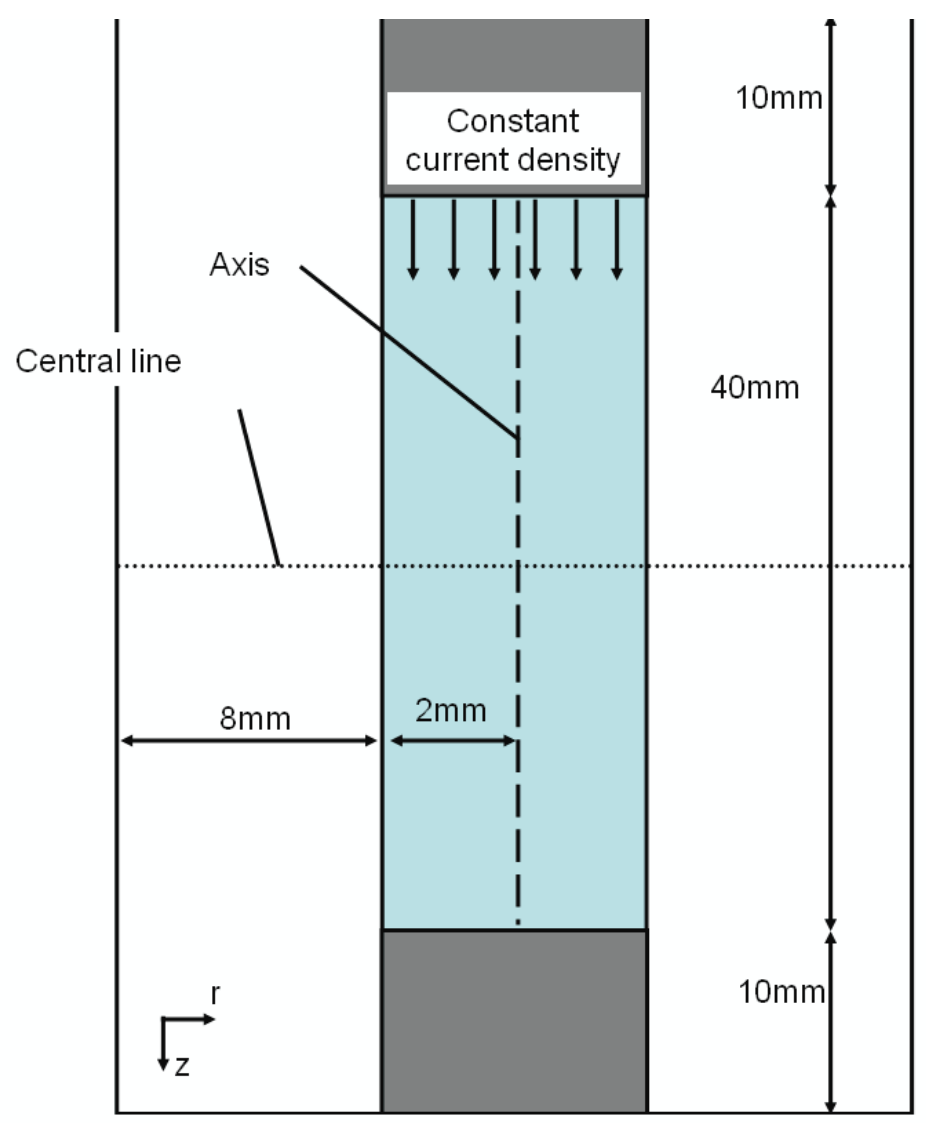

Figure 1: Cylinder with a constant current density distribution 


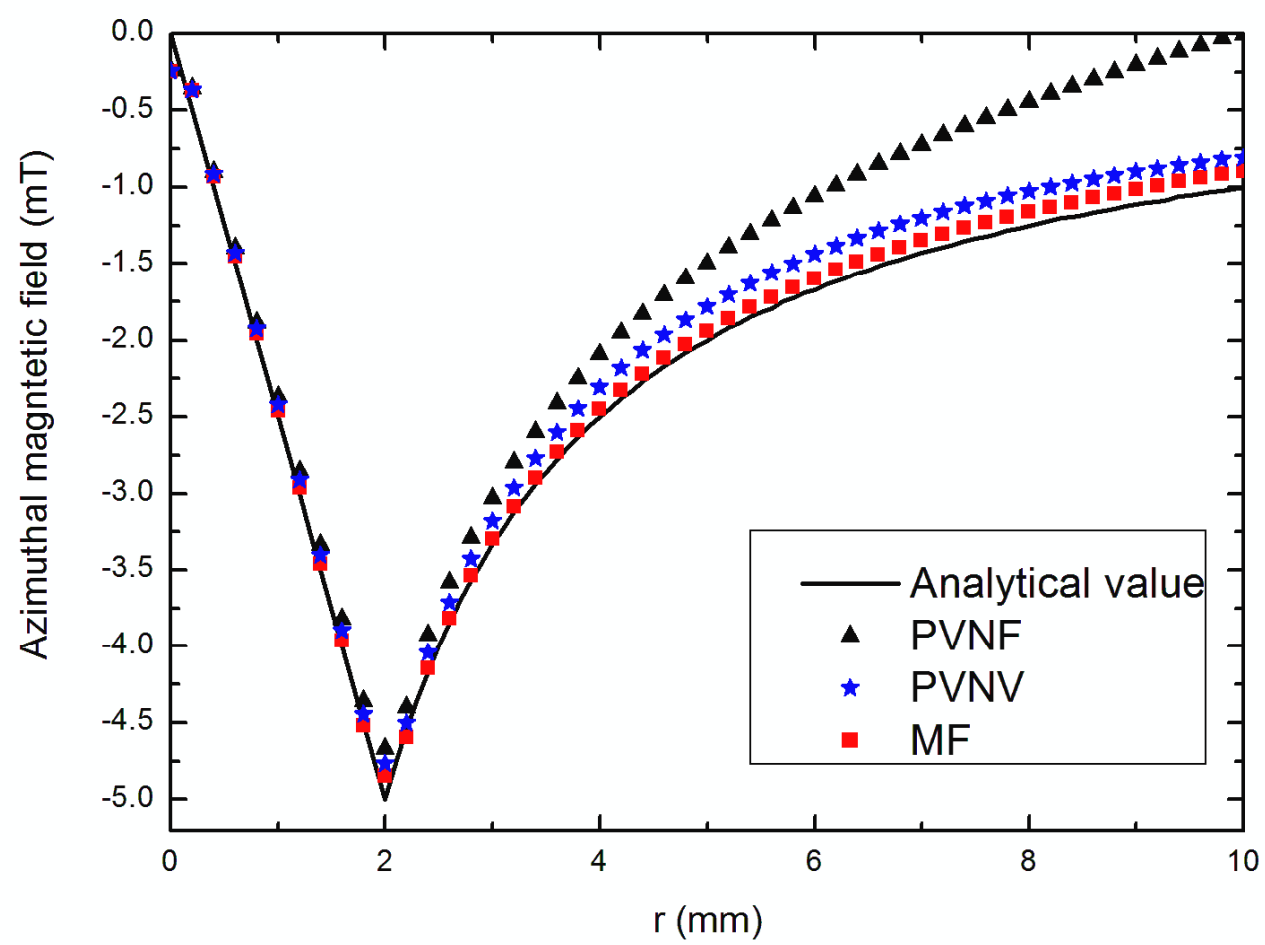

Figure 2: Azimuthal magnetic field in the middle plane of the cylinder 

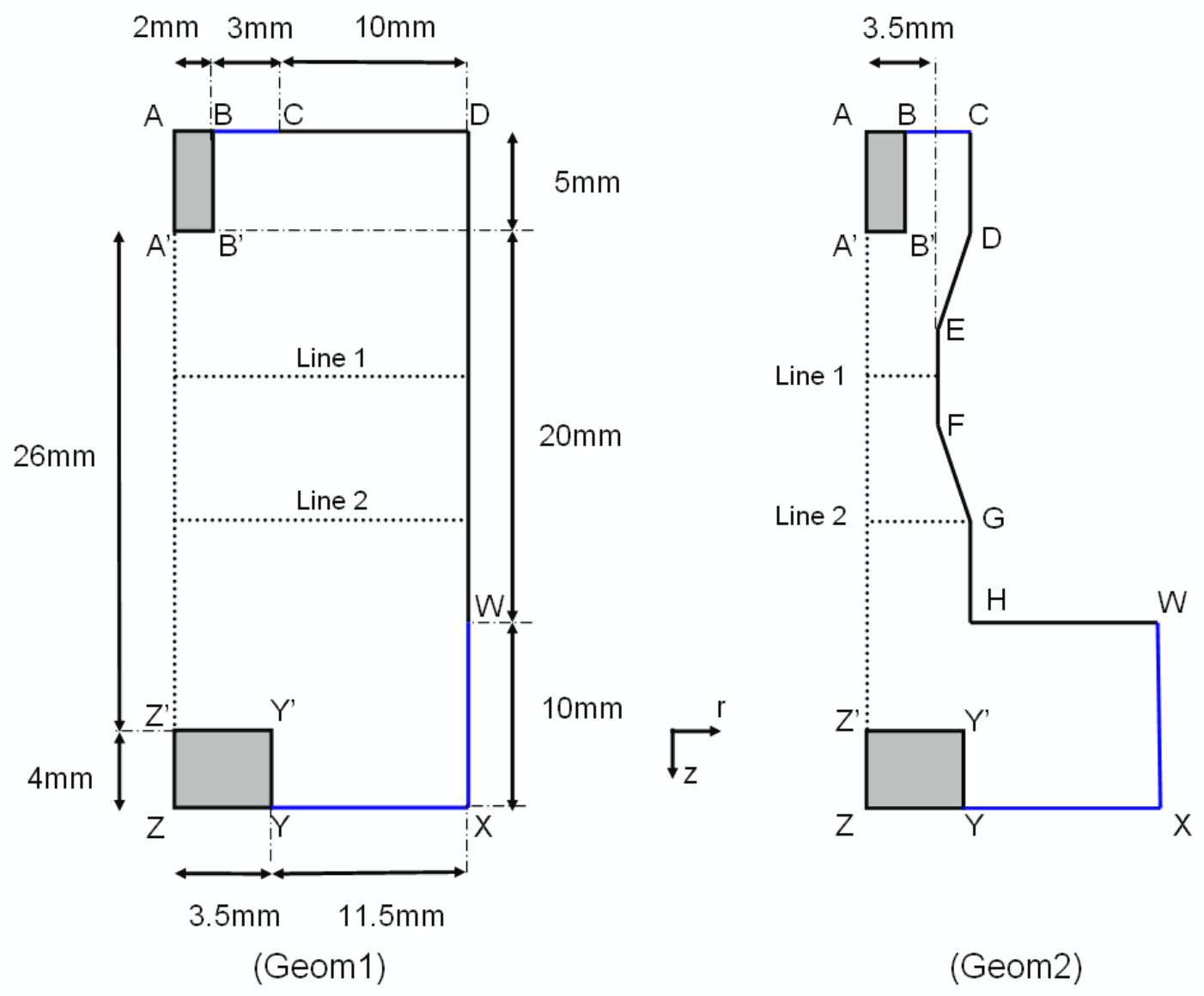

Figure 3: The two geometries studied:

(Geom1) Free expansion geometry. (Geom2) Constricted geometry. 


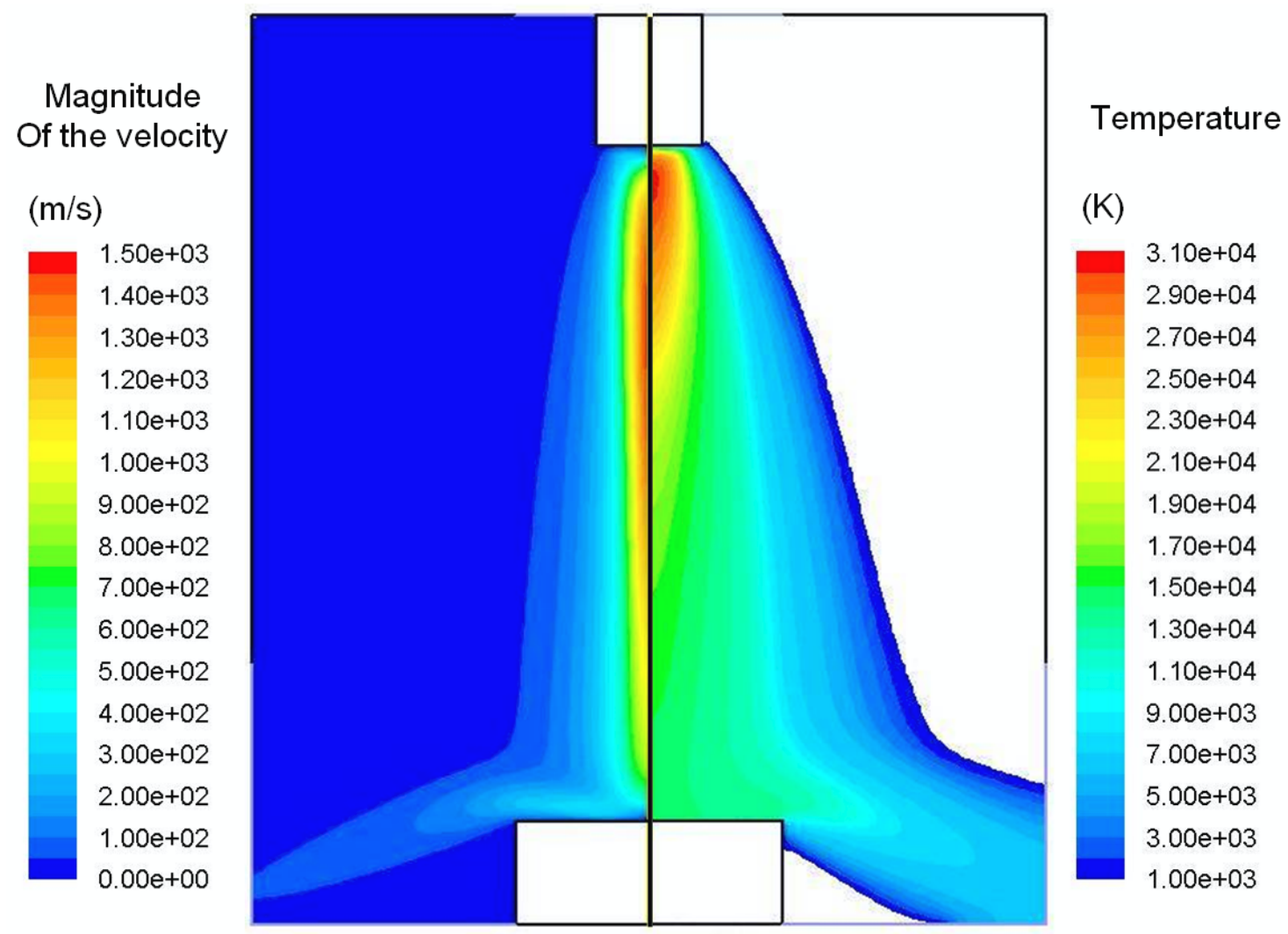

Figure 4: Fields of temperature (left) and velocity magnitude (right) for case 1-1 


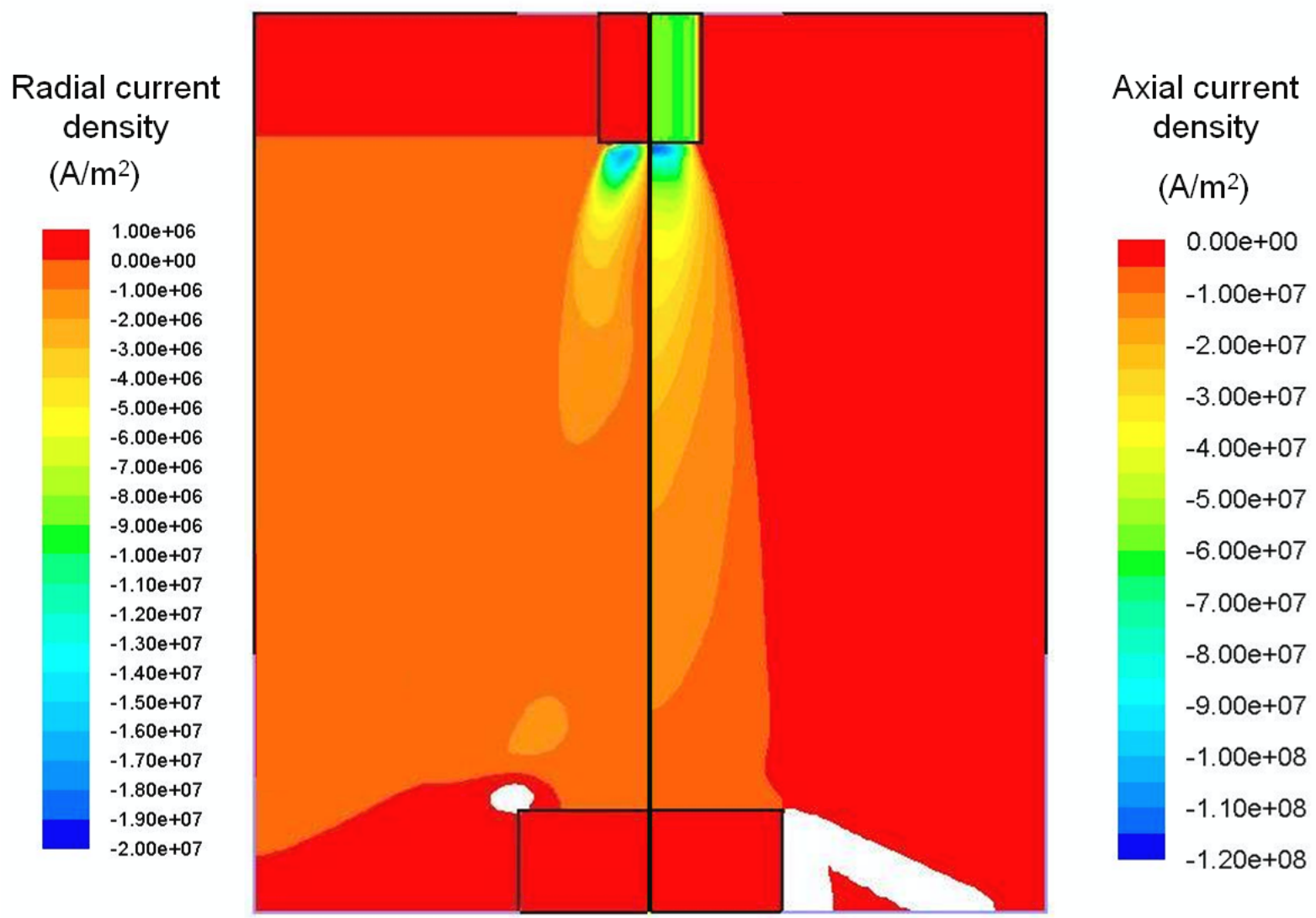

Figure 5: Fields of current density components for case 1-1 


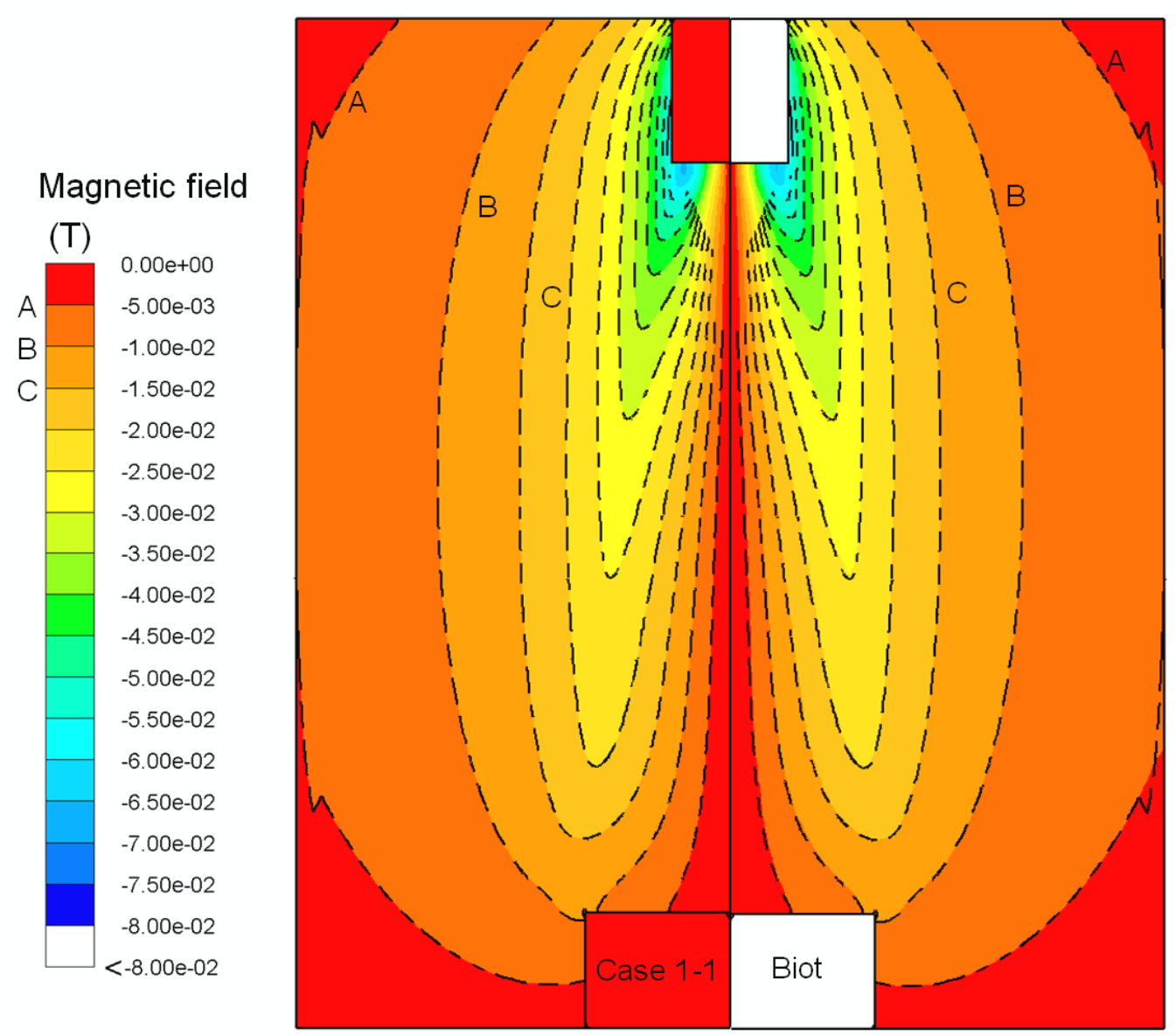

Figure 6: Comparison of magnetic field obtained by the mixed formulation (case 1-1) and the full Biot \& Savart Calculation in case of Geom1. 


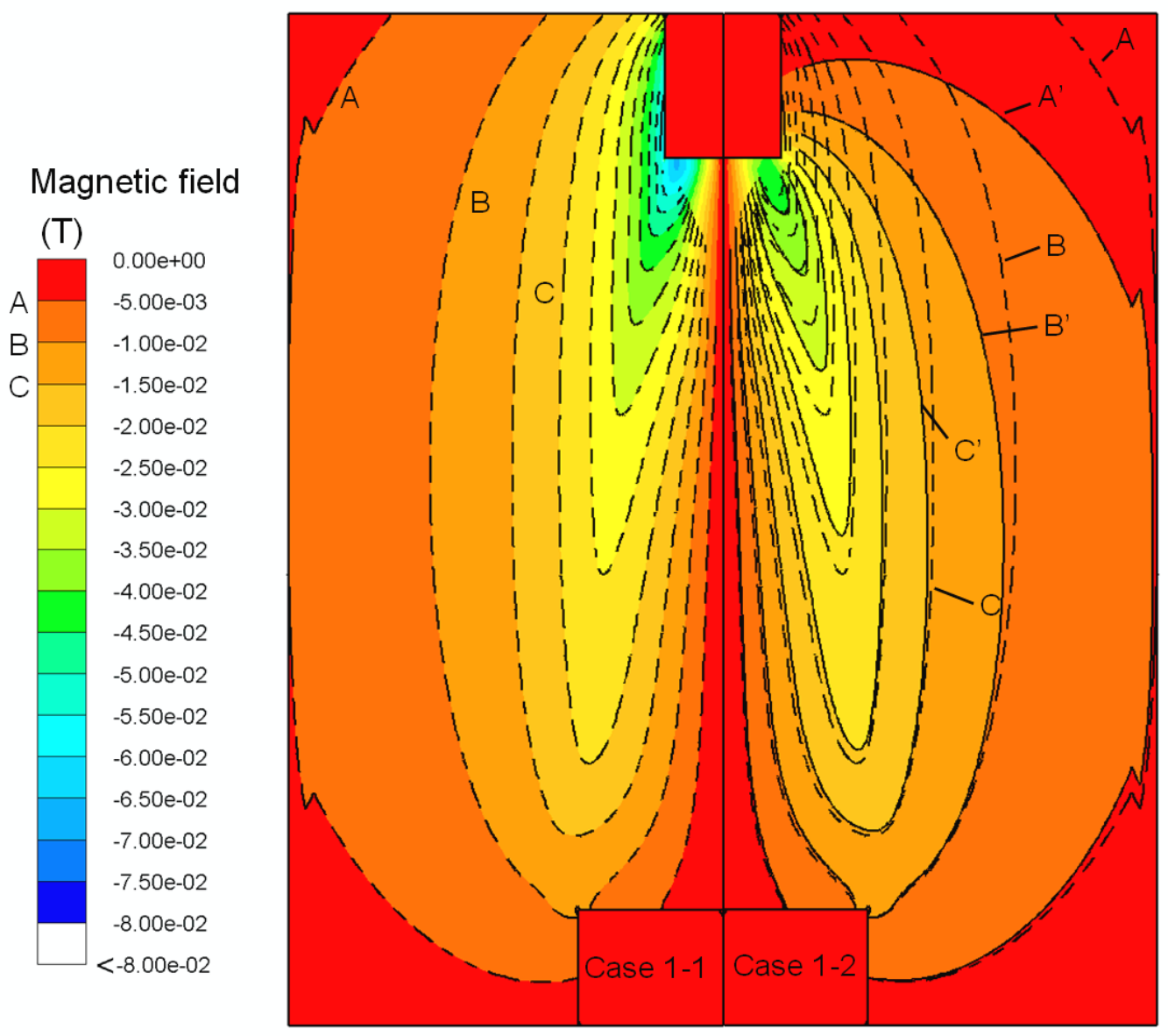

Figure 7: Comparison of azimuthal component of the magnetic field obtained in case 1-1

(M.F. with cathode) and case 1-2 (MF without cathode) 


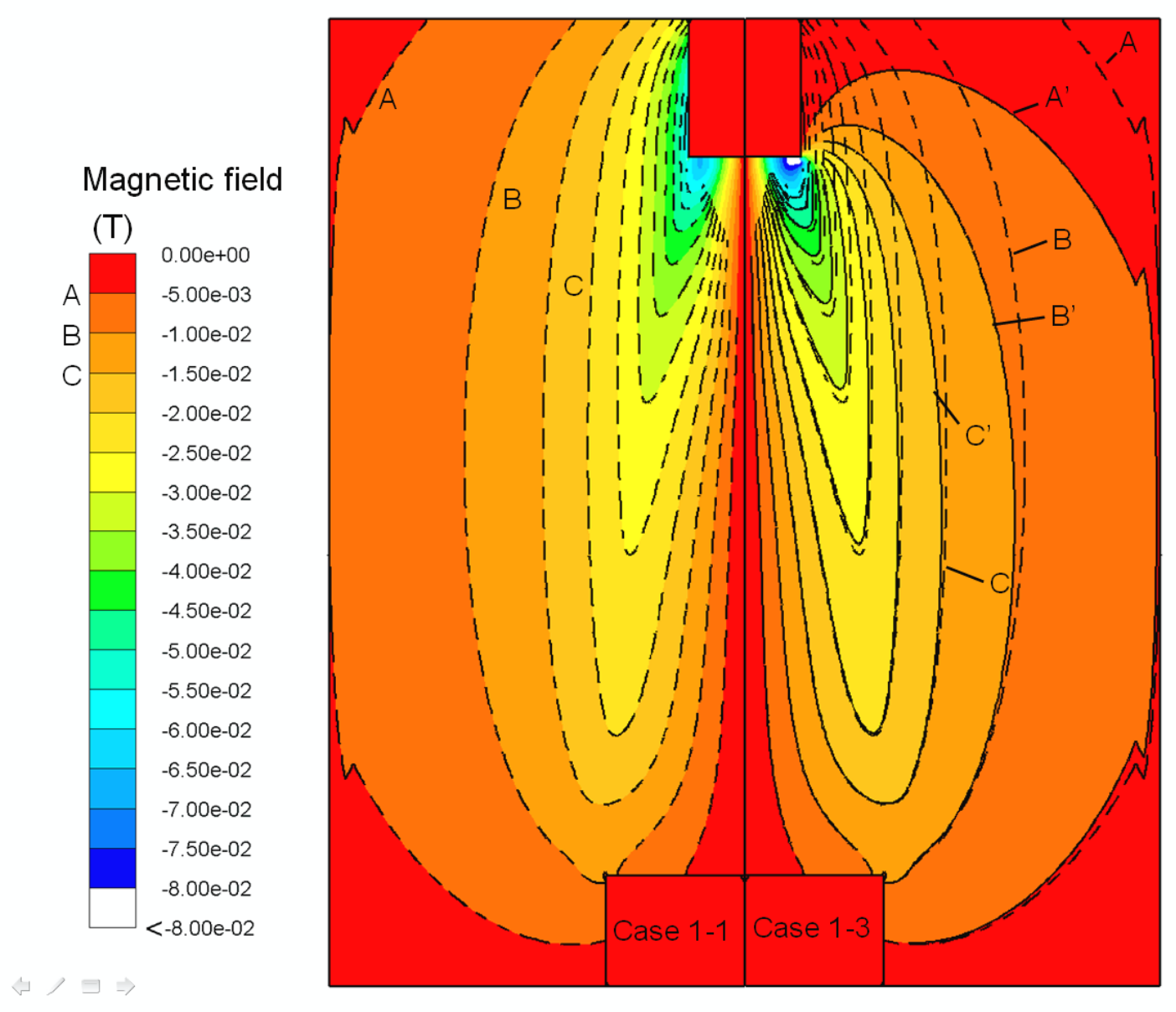

Figure 8: Comparison of azimuthal component of the magnetic field obtained in case 1-1 and case 1-3 (M.F. With null flux on the arc attachment locations: anode and cathode) 


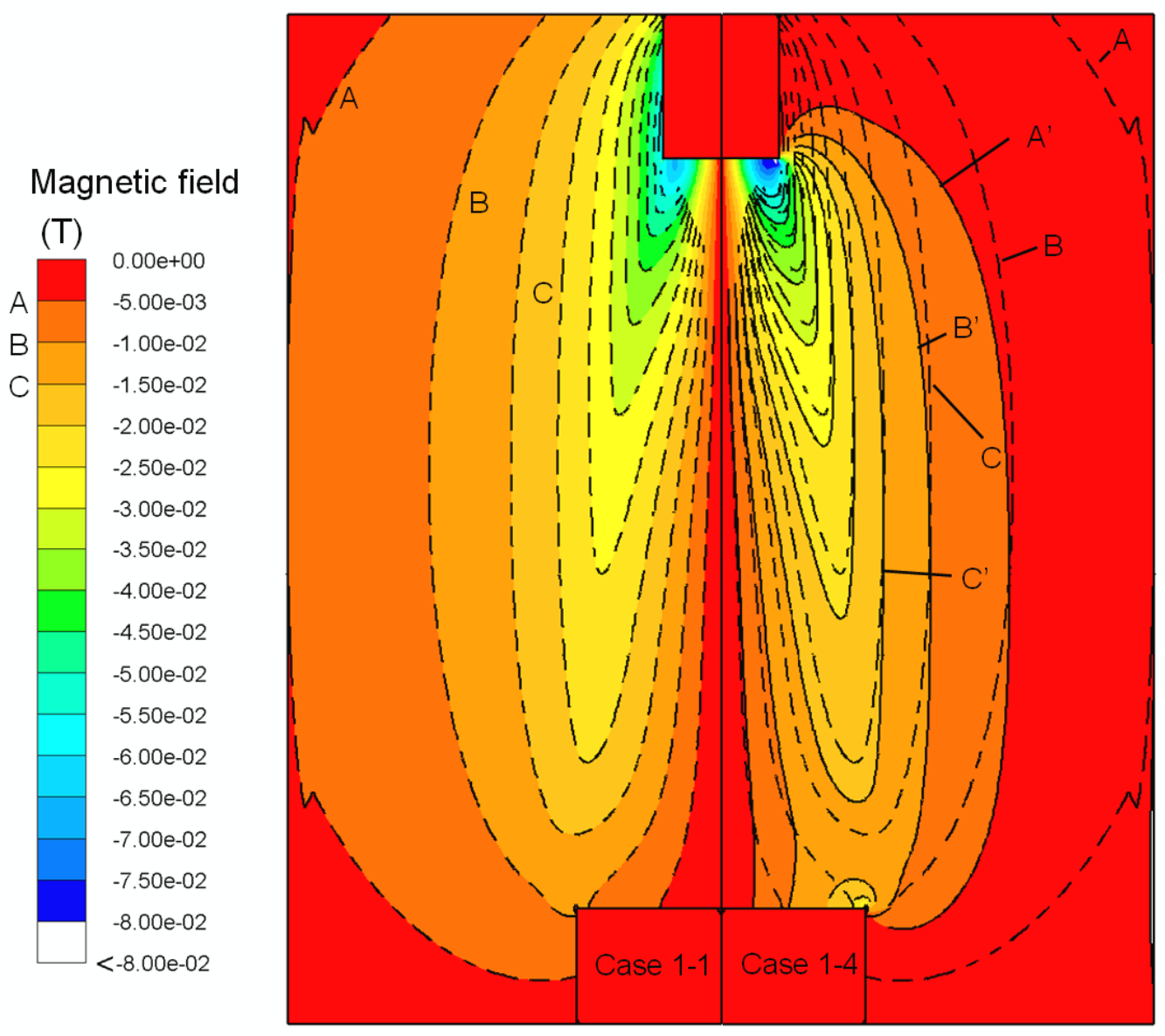

Figure 9: Comparison of azimuthal component of the magnetic field obtained in case 1-1 and case 1-4 (P.V.N.F.) case 1-4 (P.V.N.F.) 

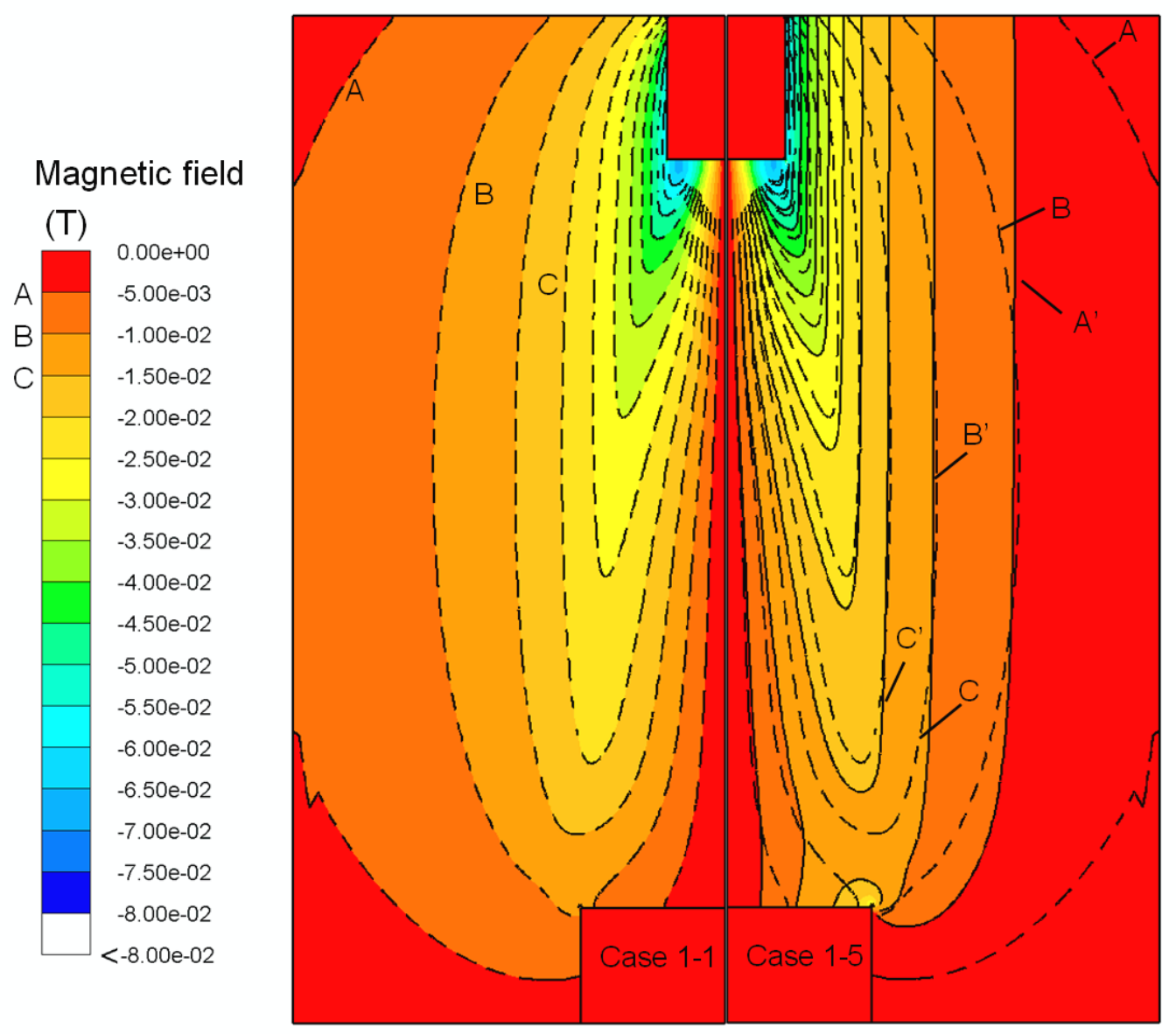

Figure 10: Comparison of azimuthal component of the magnetic field obtained in case 1-1 and case 1-5 (P.V.N.F.) 


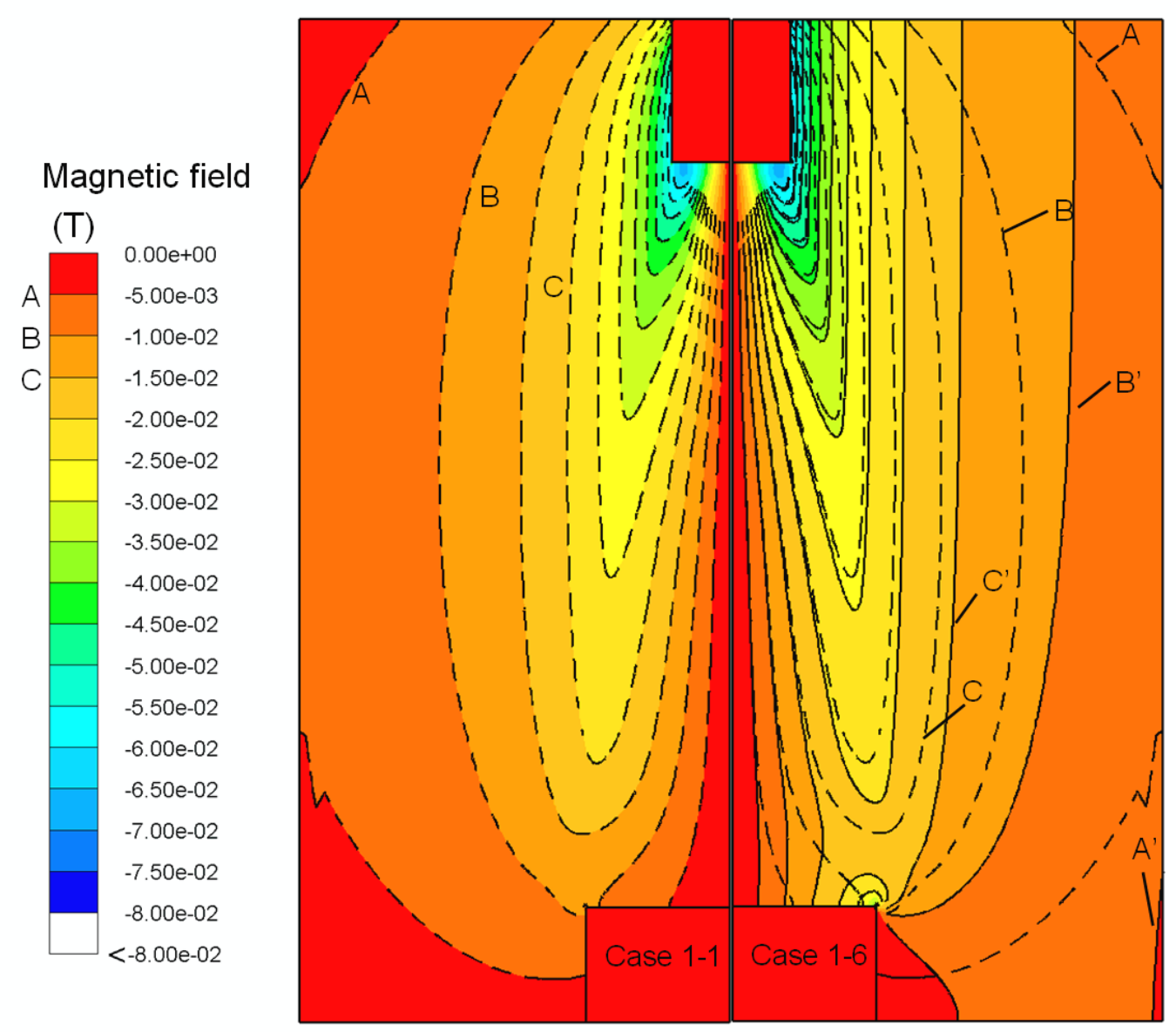

Figure 11: Comparison of azimuthal component of the magnetic field obtained in case 1-1 and case 1-6 (P.V.N.V.) 

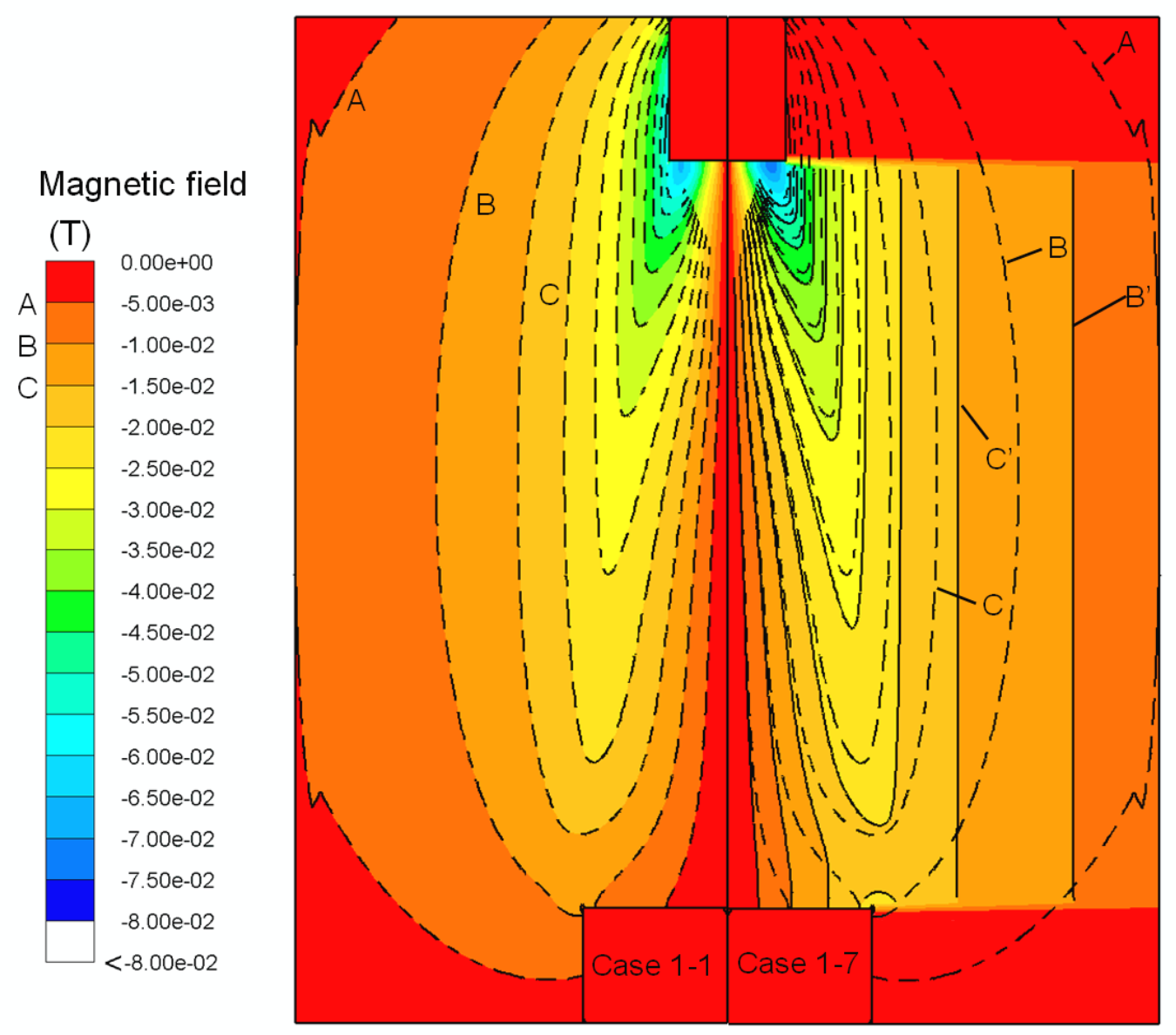

Figure 12: Comparison of azimuthal component of the magnetic field obtained in case 1-1 and case 1-7 (M.A.) 

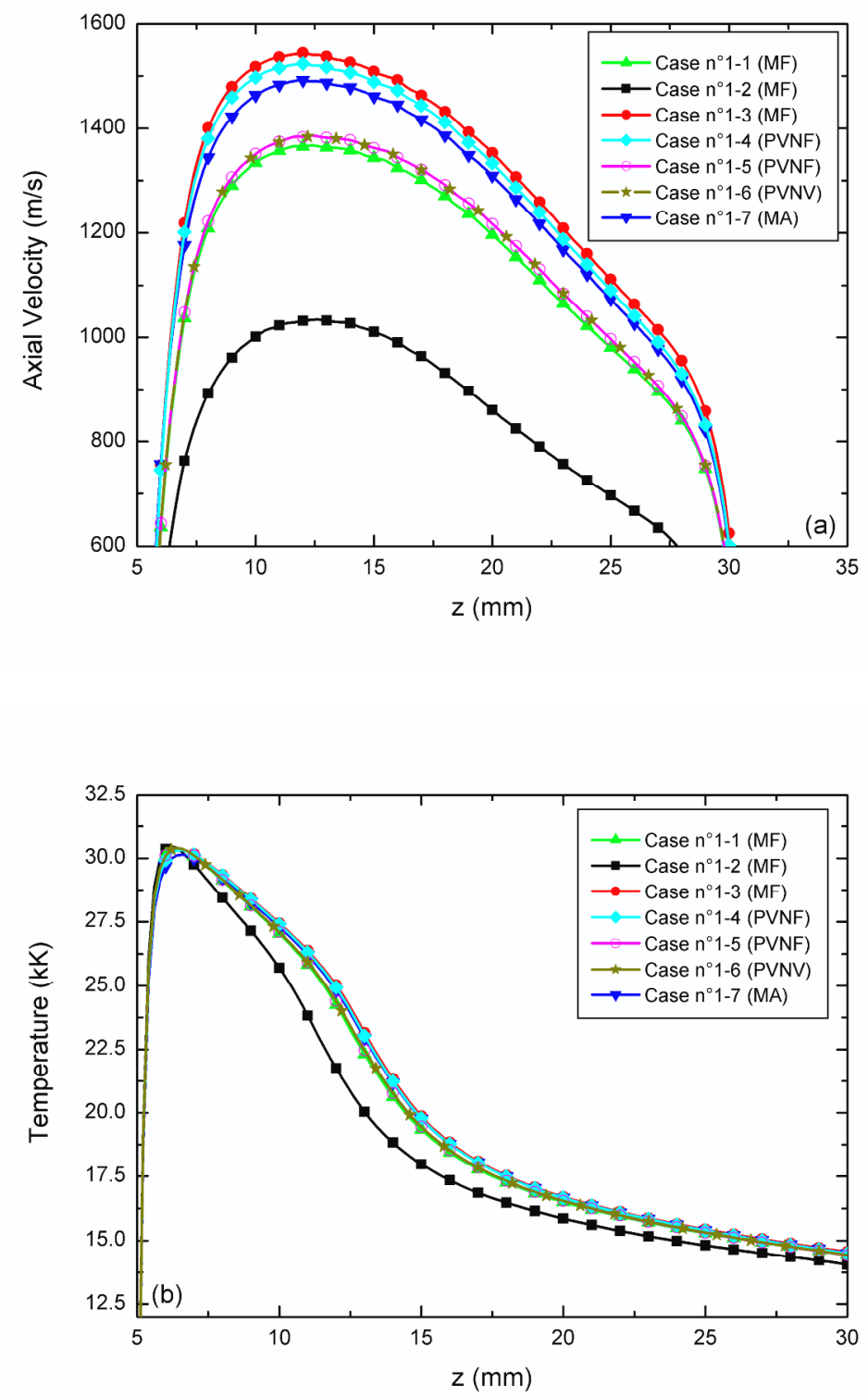

Figure 13 (a\&b): Axial velocity and temperature in Geom1. 

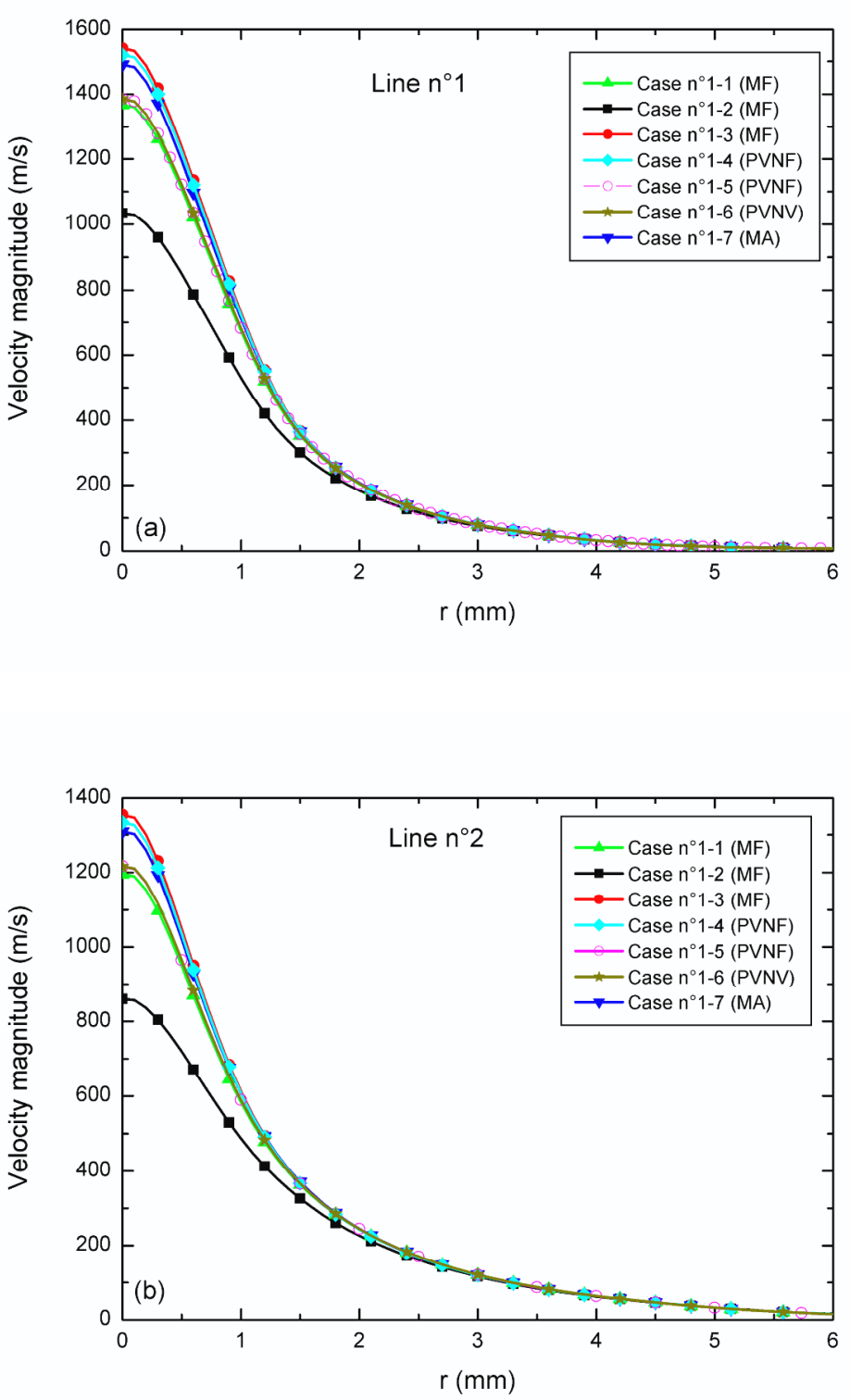

Figure $14 \mathrm{a} \& \mathrm{~b}$ : Velocity profiles along lines 1 and line 2

(Respectively $\mathrm{z}=12.5 \mathrm{~mm}$ and $20 \mathrm{~mm}$ ) 

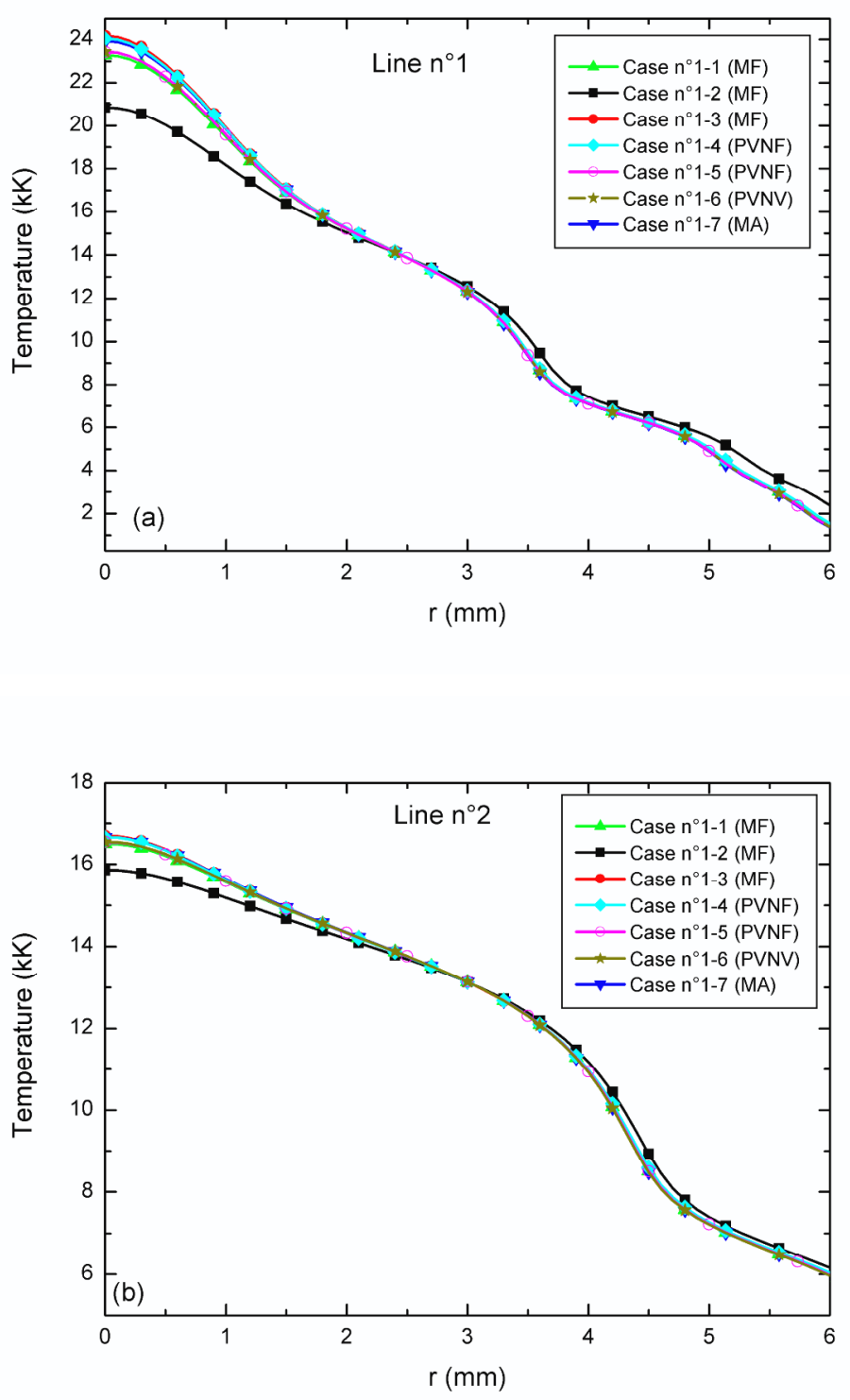

Figure $15 \mathrm{a} \& \mathrm{~b}$ : Temperature profiles along lines 1 and line 2 (Respectively $\mathrm{z}=12.5 \mathrm{~mm}$ and $20 \mathrm{~mm}$ ) 


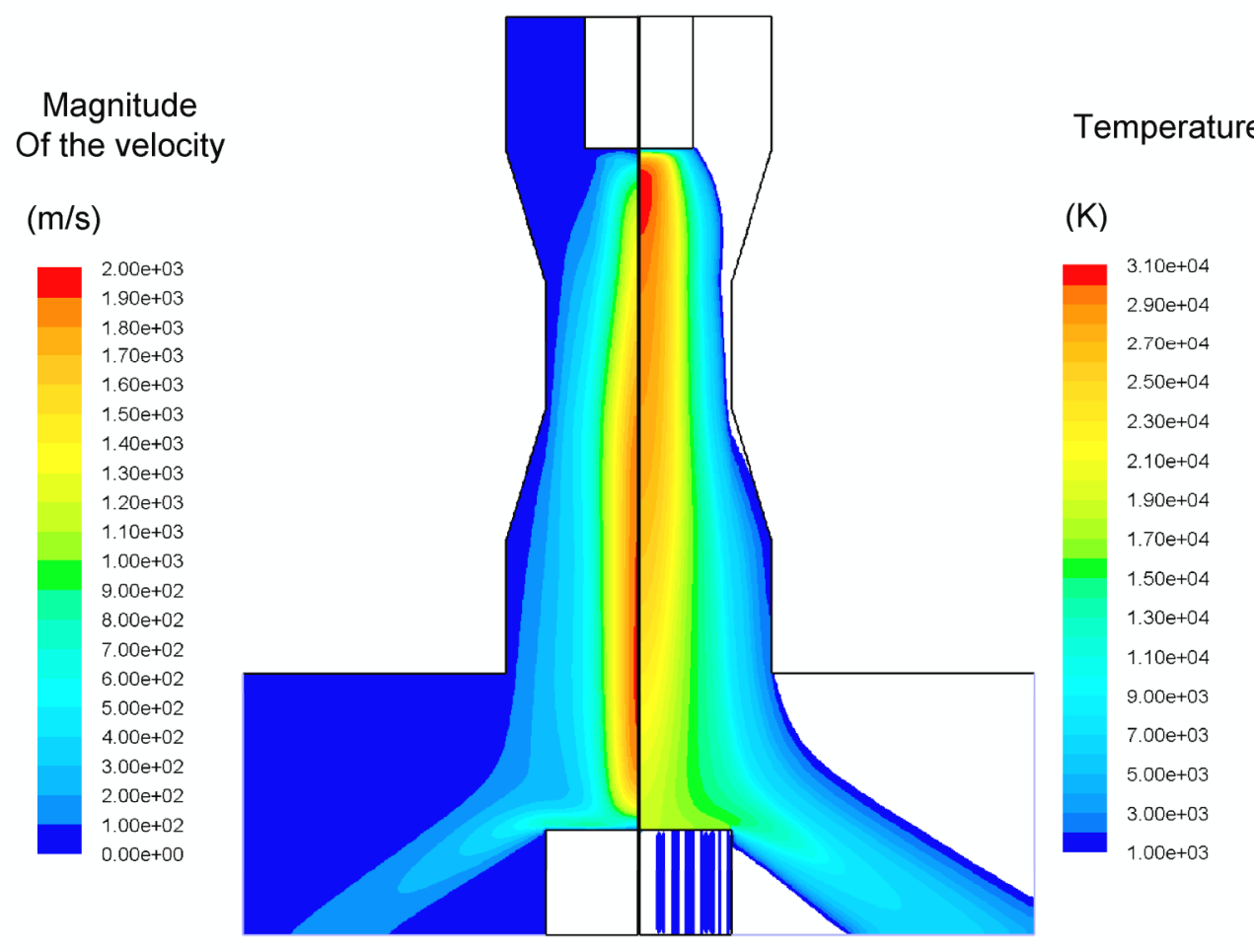

Figure 16: Fields of temperature and of the magnitude of the velocity for case 2-1 (M.F. With cathode) 


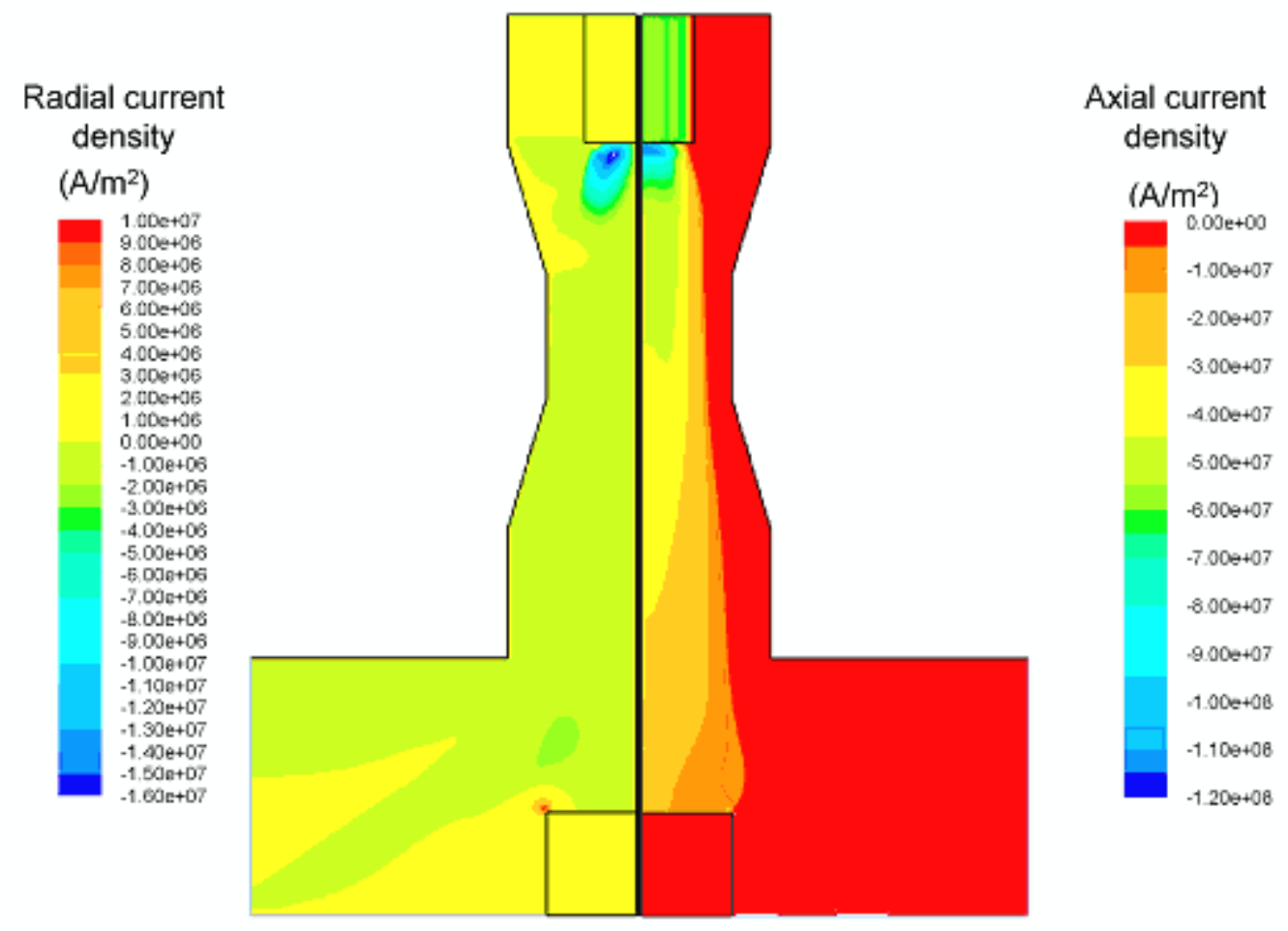

Figure 17: Current density components for case 2-1 


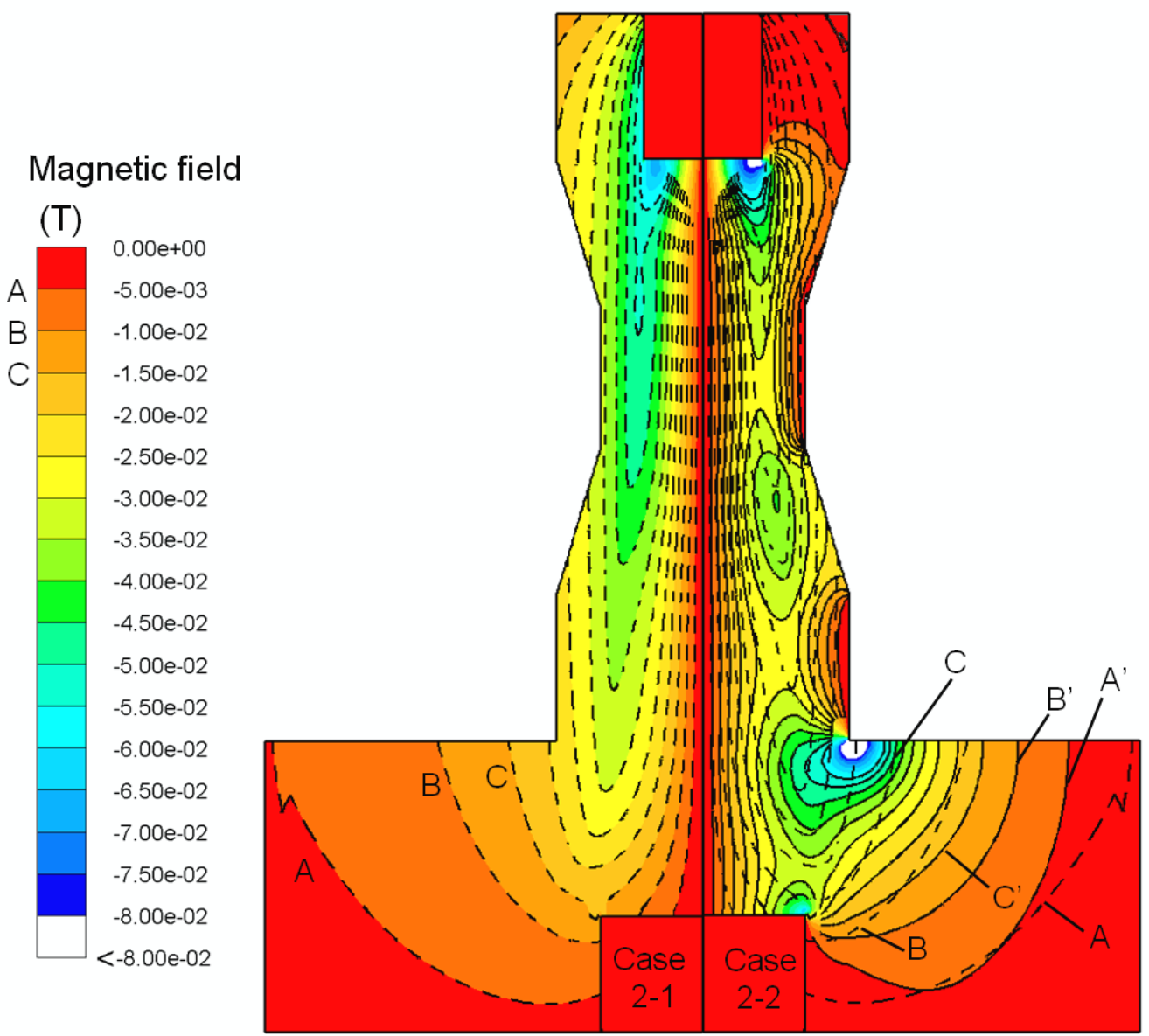

Figure 18: Comparison of azimuthal component of the magnetic field obtained in case 2-1 (M.F. with cathode) and case 2-2 (P.V.N.F. without cathode) 


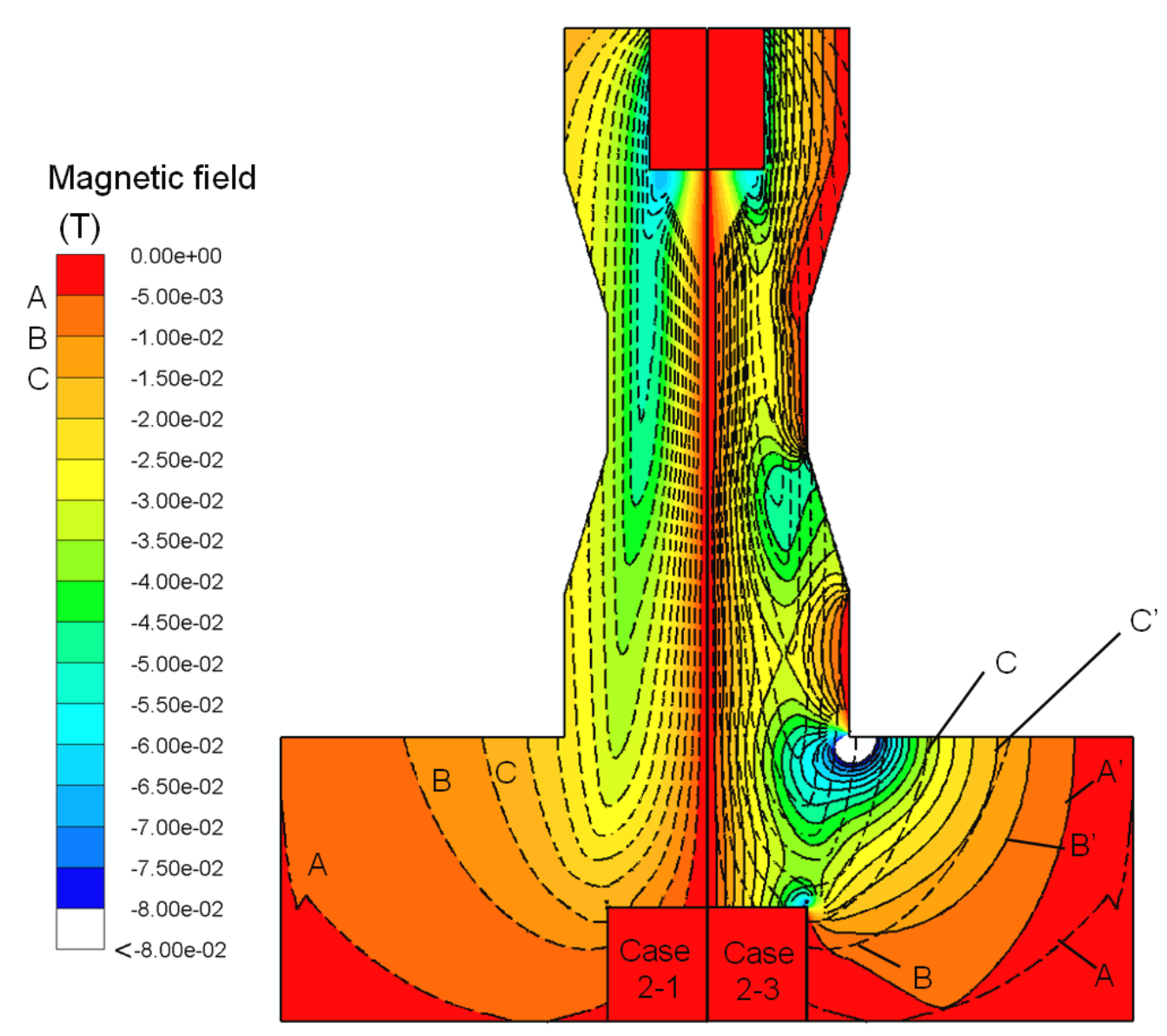

Figure 19: Comparison of azimuthal component of the magnetic field obtained in case 2-1 (M.F. with cathode) and case 2-3 (PVNF with cathode) 


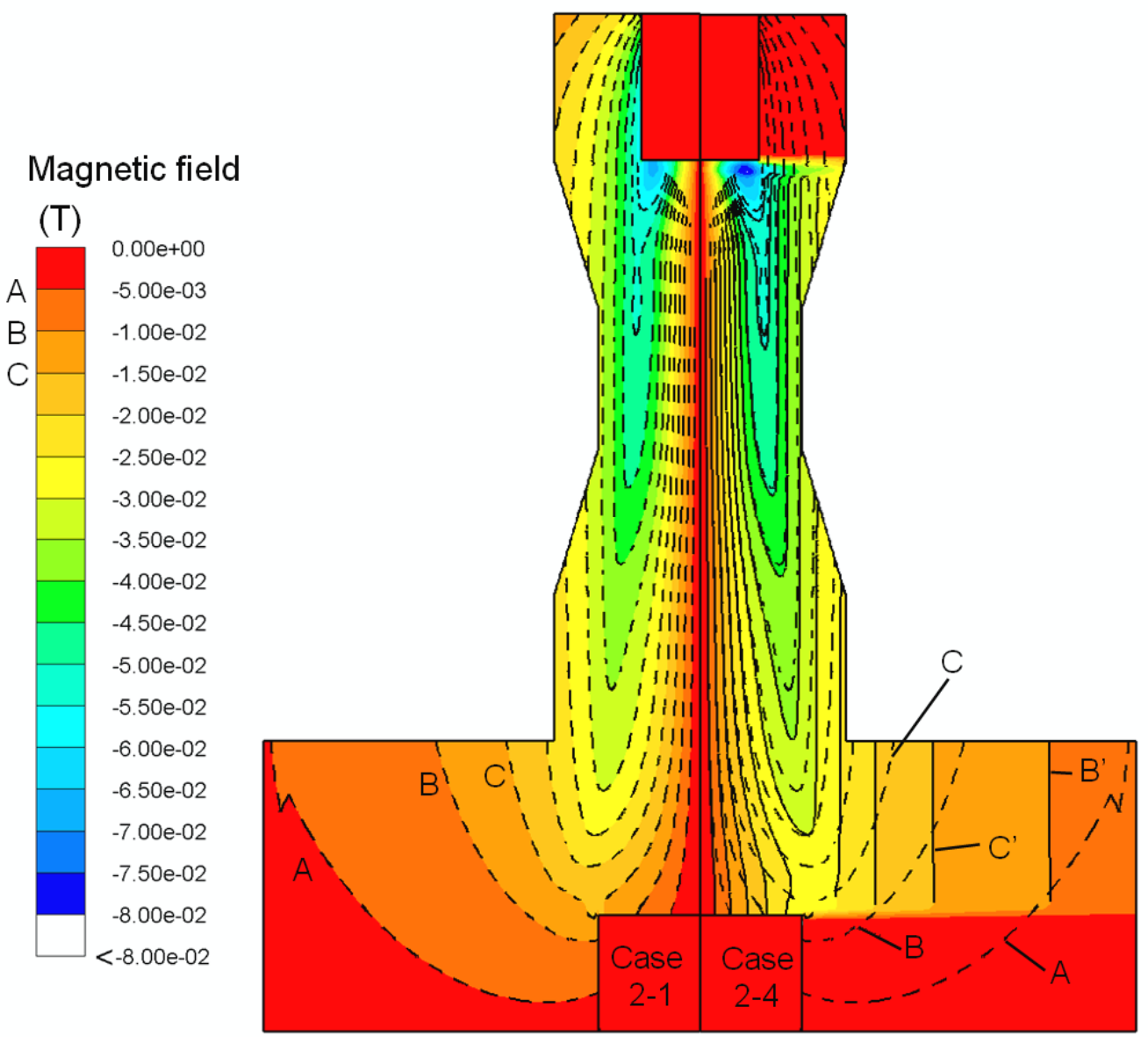

Figure 20: Comparison of azimuthal component of the magnetic field obtained in case 2-1 (M.F. with cathode) and case 2-4 (MA) 

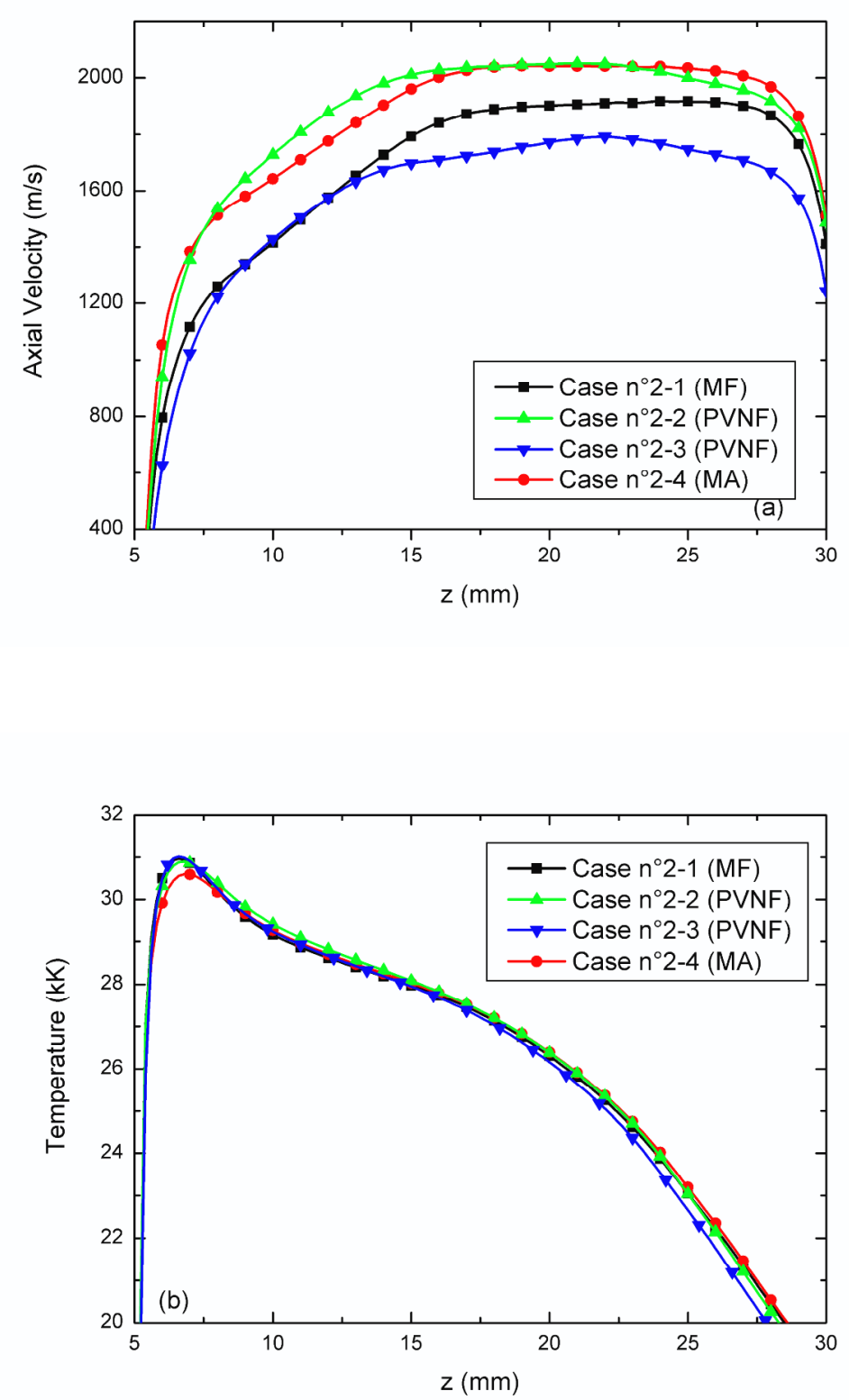

Figure 21a\&b: Evolution of the axial velocity (a) and or the axial temperature (b) in geom2. 


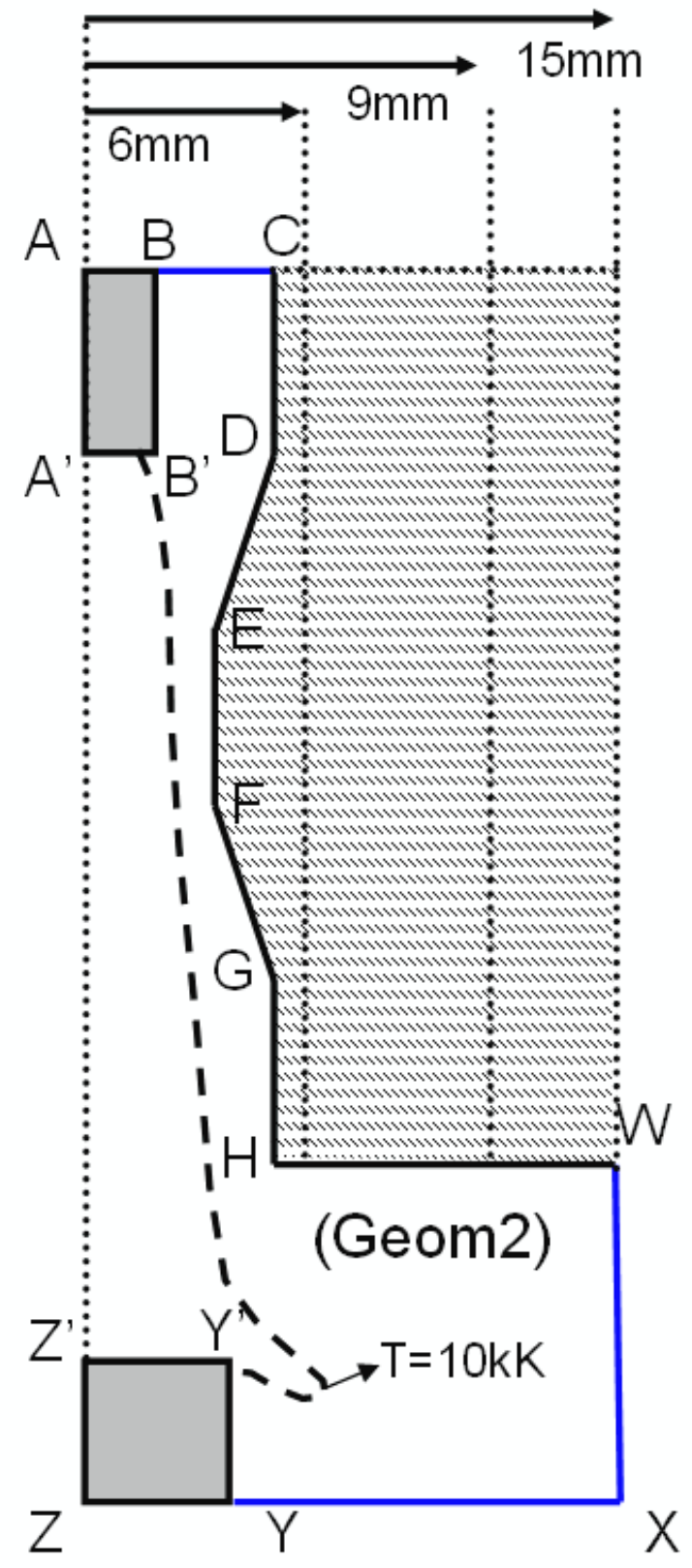

Figure 22 : Geom2 with hatched extended domain 


\section{Magnetic field}
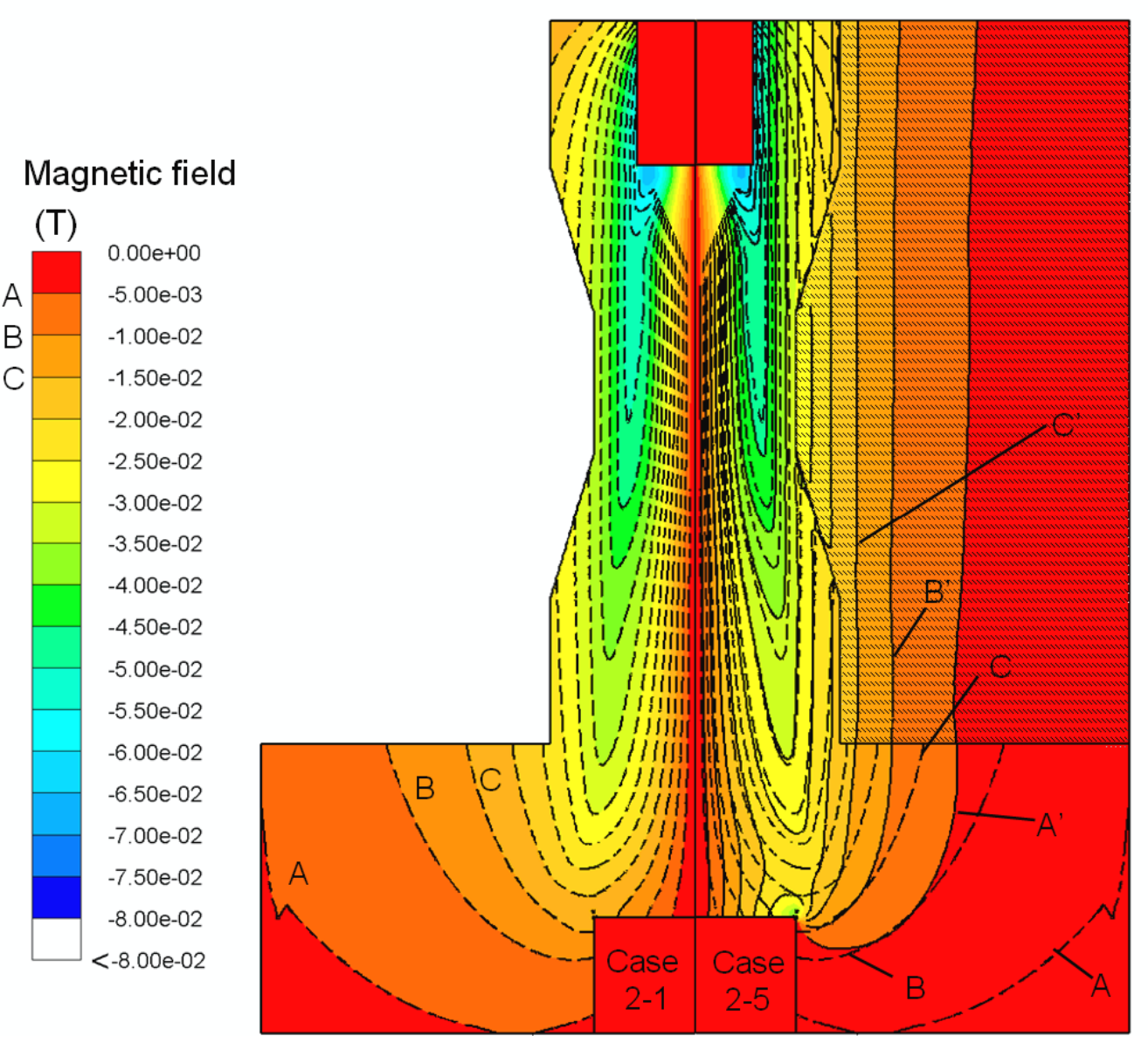

Figure 23 : Comparison of azimuthal component of the magnetic field obtained in case 2-1 (M.F. with cathode) and case 2-5 (PVNF extended domain 15mm) 


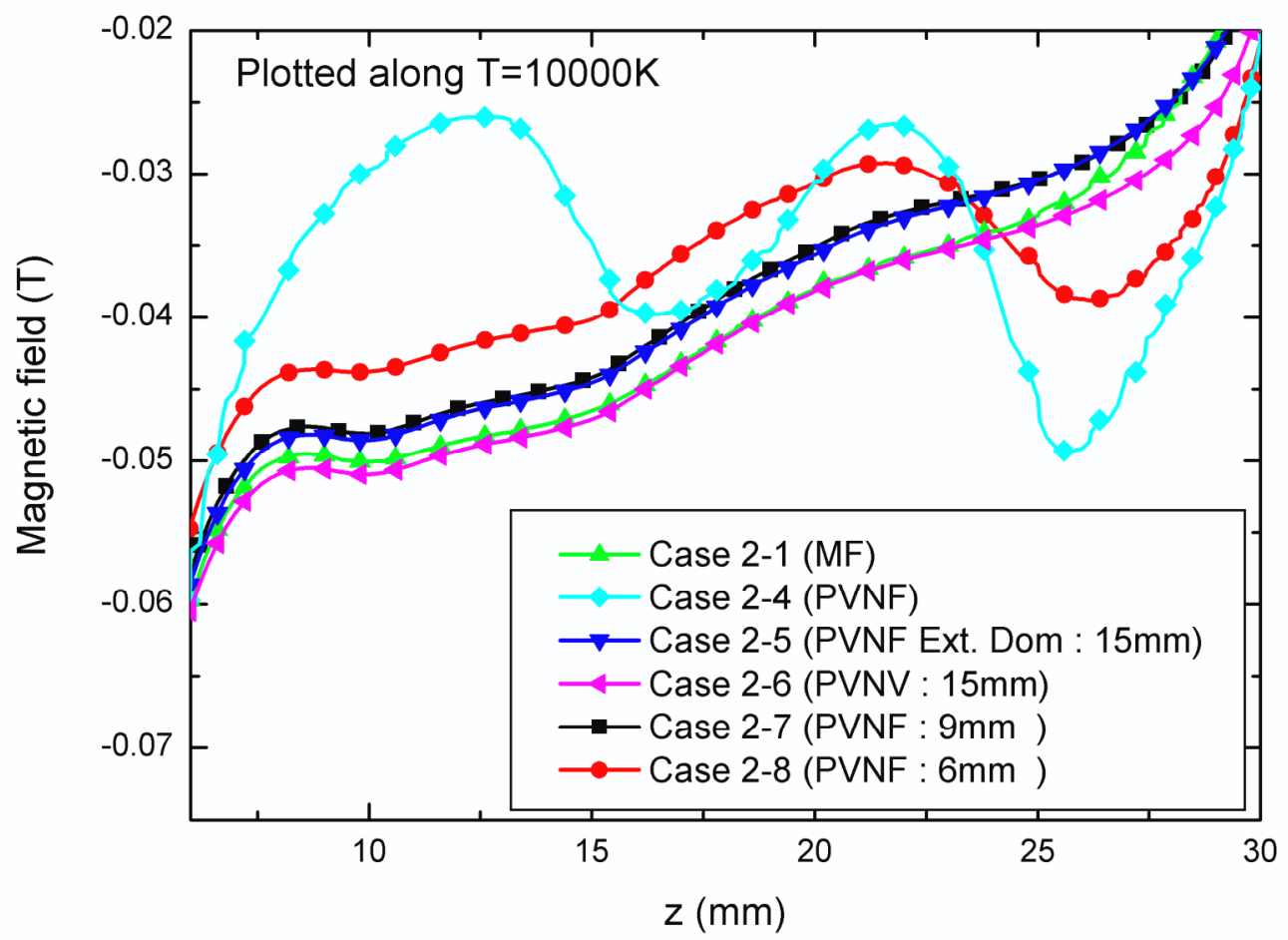

Figure 24: Comparison of the magnetic field along isotherm $10 \mathrm{kK}$, depending on the size of the extended domain and the formulation. 


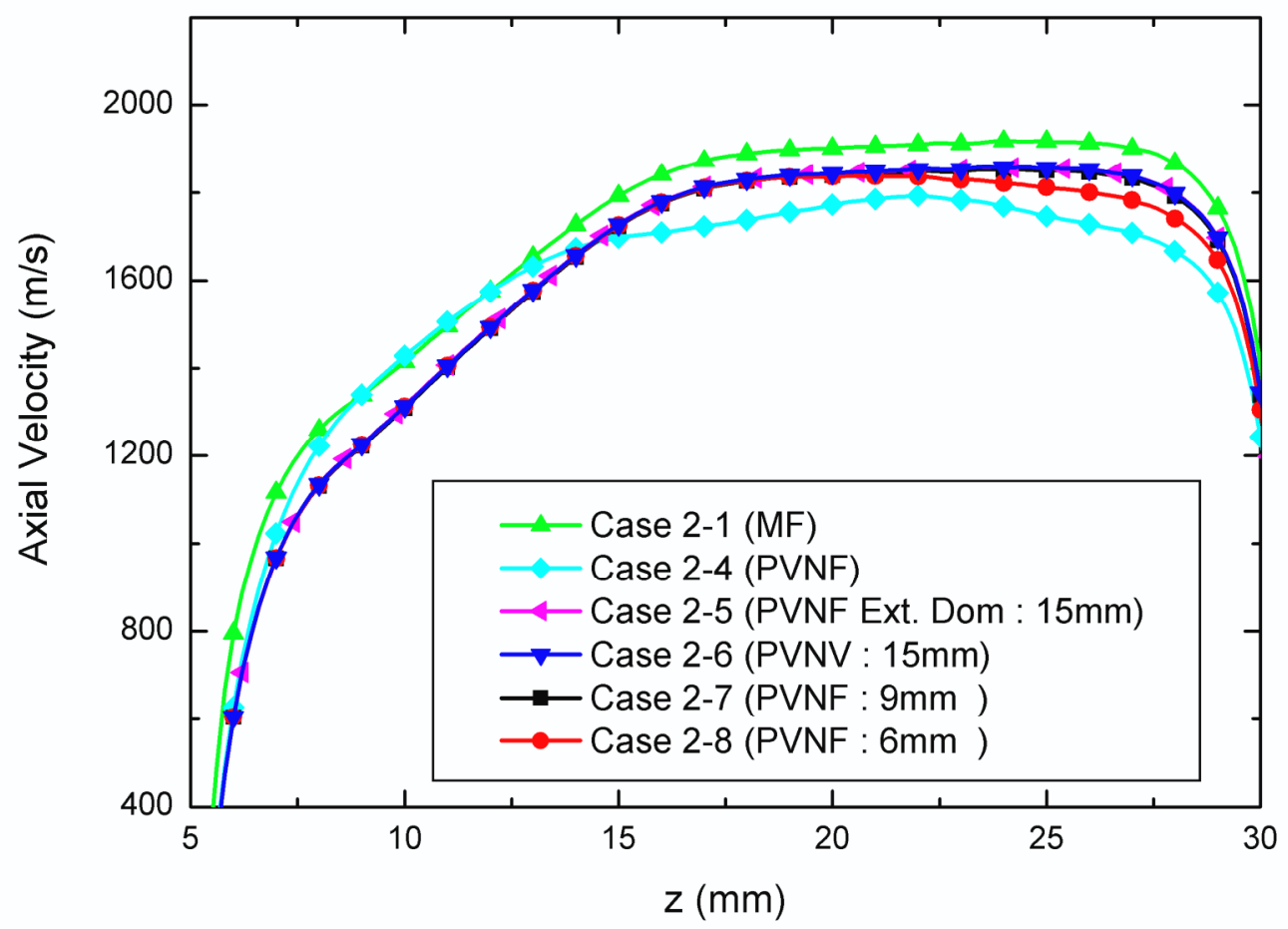

Figure 25: Evolution of the axial velocity in geom. 2 depending on the size of the extended domain and the formulation. 